\title{
Pulsar electrodynamics: Relativistic kinetic theory of radiative plasmas - collective phenomena and their radiation
}

A. A. da Costa, D. A. Diver, E. W. Laing, C. R. Stark and L. F. A. Teodoro

This is the author's final version of this article. Published version (c) American Physical Society

da Costa, A.A., et al. 2011. Pulsar electrodynamics:

relativistic kinetic theory of radiative plasmas - collective phenomena and their radiation. Physical Review D. 83(2): 023013. doi: 10.1103/PhysRevD.83.023013

The final published version is available from doi: https://doi.org/10.1103/PhysRevD.83.023013 


\title{
Pulsar electrodynamics: relativistic kinetic theory of radiative plasmas - collective phenomena and their radiation
}

\author{
A. A. da Costa ${ }^{\dagger,}$, D. A. Diver ${ }^{\ddagger}$, E. W. Laing ${ }^{\ddagger}$, C. R. Stark ${ }^{\ddagger}, \diamond$ and L. F. A. Teodoro ${ }^{\ddagger}, *$ \\ $\dagger$ Secção de Telecomunicações, Departmento de Engenharia Electroténica e Computadores \\ Instituto Superior Técnico-UTL, 1049-001 Lisboa, Portugal \\ $\ddagger$ SUPA, School of Physics and Astronomy, Kelvin Building \\ University of Glasgow, Glasgow, G12 8QQ, Scotland, UK \\ $\diamond$ School of Mathematics and Statistics, University of St Andrews, \\ Mathematical Institute, St Andrews, KY16 9SS, Scotland, UK \\ * BAER, Space Sciences and Astrobiology Division, MS 254-3 \\ NASA Ames Research Center, Moffett Field, CA 94935-1000, USA
}

(Dated: November 12, 2010)

\begin{abstract}
The classical modelling of radiation by accelerated charged particles in pulsars predicts a cut-off in photon energy at around $25 \mathrm{GeV}$. Whilst this is broadly consistent with observations, the classical treatment is not self-consistent, and cannot be extended to explain the rare high-energy detections of photons in the 100s of $\mathrm{GeV}$ range. In this paper we revisit the theoretical modelling of high-energy radiation processes in very strong electromagnetic fields, in the context of both single particles and collective plasmas. There are no classical constraints on this description. We find that there is indeed a critical energy of around $50 \mathrm{GeV}$ that arises naturally in this self-consistent treatment, but rather than being a cut-off, this critical energy signals a transition from radiation that is classical to a quasi-quantum description, in which the particle is able to radiate almost its total energy in a single event. This new modelling therefore places pulsar radiation processes on a more secure physical basis, and admits the possibility of the production of $\mathrm{TeV}$ photons in a self-consistent way.

PACS numbers: 97.60.Gb
\end{abstract}

\section{INTRODUCTION}

Pulsar magnetospheres are composed of magnetised electron-positron (pair) plasmas characterised by ultra strong magnetic fields and broadband electromagnetic emission, covering all frequency spectra, from radio to hard $\gamma$-ray radiation. However there is a cut-off for observed radiated photons at the $20 \mathrm{GeV}$ range $[1,2]$.

Various geometric models have been proposed that are specific to particular objects (mainly the Crab) which do not explain the cut-off from first principles, but instead assume that it exists, and propose models that recover the consequent power spectrum (for example [35]). Whilst this approach has its merits in interpreting the data, it is unable to explain the intrinsic origin of this abrupt fall of the $\gamma$-ray radiated power with frequency, and this disappearance in the $\mathrm{TeV}$ range. Instead we must address directly the dynamics of the pulsar magnetosphere, as it was done in the Higgins \& Henriksen's seminal papers [6,7], requiring improvement, since they used a classical single particle approach without going beyond the classical description, and they do not take into account properly the required influence of the radiation in the particle motion as we do in this paper.

More recently there have been further attempts to model the global pulsar electrodynamics from first principles, in order to tackle a particular object, or to describe an overall geometry. For example, [8] presents a numeri- cal simulation of the general rotating magnetic dipole in $3 \mathrm{D}$, from the pulsar surface to the light cylinder; this calculation incorporates the ideal MHD description of the evolution of the imposed rotating dipole, and so is not able to address the fundamental radiation physics for ultra relativistic single particles. Tang and co-workers [9] have applied parametric fitting of high-energy radiation mechanisms combined with geometric assumptions to obtain good fits to the high-energy spectral components of the Crab in a specialised application. Force-free global geometries for pulsar electrodynamics have also been investigated [10] as possibly revealing the magnetospheric radiation source position, but without detailing how particles are accelerated to such positions. Finally, combining the global magnetic environment of the pulsar with the escaping high-energy wind was the object of the numerical simulations of Wada and co-authors [11]; this article cites the electric induction as the accelerator, but does not investigate in detail the actual acceleration of particles under the derived fields, nor does this numerical work account for the collective contributions to the fields by such particle populations.

Whilst these papers represent genuine progress in describing the global pulsar environment, there is still the need to address the essential physics of the acceleration mechanism itself, and the consequences for both the magnetosphere and the radiation emission of the collective contribution made by cohorts of accelerated charges. 
These twin objectives are at the core of this paper.

The presence of an electron-positron plasma in pulsar magnetospheres is well established since the Goldreich \& Julian's early work [12] for the aligned model, and later generalized to the non-aligned model by Mestel [13, 14] and Cohen \& Toton [15]. This plasma is assumed to leave the star from rest, and accelerated by a low frequency EM field, a combination of the star dipolar EM field, described by the Deutsch Model [16] and the one generated by several processes occurring in the star and which will be identified later, a very difficult functional problem.

The magnetospheric plasma is assumed to be collisionless, in the Vlasov sense that is, the typical collision frequency $\nu$ for all particle interactions (including annihilation) is much less than the plasma frequency $\omega_{p}: \omega_{p} \gg \nu$ and the plasma remains dominated by the influence of the common fields. For simplicity here, we shall assume that the typical 'relaxation time' will be the plasma period. Given the magnitude of the EM fields, a cold plasma approach is an acceptable one, with a close analogy with the single (test) particle approach, due to the formal equivalence between the Vlasov equation and standard orbit theory [17], as seen in [6, 7, 18-24].

Following the single particle approach, the dynamics of pulsar magnetosphere assumes the classical description, and must take into account radiative effects. As accelerated charged particles radiate, the higher the particle energy, the higher the frequency of the radiated photons, and the particle power radiated, the Larmor power, creating a braking force, the Dirac force. Such force must be taken into account in the equation of motion, (contrary to what was done in $[6,7]$ ) and this is done classically substituting the usual Lorentz equation by the one known as the Lorentz-Dirac equation of motion (L-DEM) [25],

$$
\dot{u}^{i}=\frac{e}{m} F^{i k} u_{k}+\chi \ddot{u}^{i}+\frac{\chi}{c^{2}}\left(\dot{u}^{j} \dot{u}_{j}\right) u^{i}
$$

with $\chi=e^{2} /\left(6 \pi \epsilon_{0} m c^{3}\right) \sim 6.27 \times 10^{-24} \mathrm{~s}$.

The L-DEM time-like term is, neglecting the Schott term $\left(\chi \ddot{u}^{i}\right)$, and using Landau \& Lifshitz [26] formulation:

$$
\begin{aligned}
\frac{\mathrm{d} \mathcal{E}}{\mathrm{d} t} & =e \boldsymbol{E} . \boldsymbol{v}-\frac{\chi e^{2}}{m} \gamma^{2}\left|\boldsymbol{E}_{\perp}+\boldsymbol{v} \times \boldsymbol{B}\right|^{2} \\
& \equiv P_{\text {Lor }}(\boldsymbol{r}, \boldsymbol{p}, t)-P_{\text {Dir }}(\boldsymbol{r}, \boldsymbol{p}, t),
\end{aligned}
$$

with $\mathcal{E}=\gamma m c^{2}$ the charged particle energy, $\boldsymbol{E}_{\perp}=\boldsymbol{E}-$ $(\boldsymbol{E} \cdot \boldsymbol{v}) \boldsymbol{v} / v^{2}$. $P_{\text {Lor }}(\boldsymbol{r}, \boldsymbol{p}, t)$ is the Lorentz power, the power acquired through the Lorentz force, and $P_{\text {Dir }}(\boldsymbol{r}, \boldsymbol{p}, t)$ the charged particle's total power radiated. Everything seems to work as a balance between the acquired Lorentz power and radiated Dirac power, when the L-DEM is required to substitute the Lorentz equation. This happens for $\hbar \omega_{\text {peak }}>m c^{2}$, with either $P_{\text {Dir }} \rightarrow P_{\text {Lor }}$ or $P_{\text {Dir }} \geq P_{\text {Lor }}$, with

$$
\omega_{\text {peak }} \sim 0.45 \Omega \gamma^{3},
$$

the frequency at which the peak of the radiation is emitted, following the general power spectrum of radiated photons, $P_{r}(\boldsymbol{r}, \boldsymbol{p}, \omega, t)$ as given by the following expression in the classical case, after [26] and [27], and assuming the similarity between curvature and synchrotron radiation (see Appendix B):

$$
P_{r}(\boldsymbol{r}, \boldsymbol{p}, \omega, t)=\frac{3^{5 / 2}}{8 \pi} P_{\operatorname{Dir}} \frac{\omega}{\omega_{c}^{2}} \int_{\frac{\omega}{\omega_{c}}}^{\infty} d \eta K_{5 / 3}(\eta)
$$

where [26-28]

$$
\begin{aligned}
P_{\text {Dir }} & \equiv P_{\text {Dir }}(\boldsymbol{r}, \boldsymbol{p}, t)=\chi \frac{e^{2}}{m}\left|\boldsymbol{E}_{\perp}+\boldsymbol{v} \times \boldsymbol{B}\right|^{2} \gamma^{2} \\
& \equiv P_{\mathrm{Rad}}(\boldsymbol{r}, \boldsymbol{p}, t)=\int_{0}^{\infty} \mathrm{d} \omega P_{r}(\boldsymbol{r}, \boldsymbol{p}, \omega, t) \\
\omega_{c} & =1.5 \gamma^{3} \Omega \\
\omega_{\text {peak }} & =0.29 \omega_{c} .
\end{aligned}
$$

$\Omega$ is the angular velocity related to the radius $\rho$ of the particle trajectory given by

$$
\Omega=\frac{c}{\rho}=e \frac{\left|\boldsymbol{E}_{\perp}+\boldsymbol{v} \times \boldsymbol{B}\right|}{p}
$$

which is equivalent to the angular velocity of synchrotron radiation by changing $B \Rightarrow\left|\boldsymbol{E}_{\perp}+\boldsymbol{v} \times \boldsymbol{B}\right| / c$ (see Appendix $\mathrm{B})$, as this one is

$$
\Omega=\frac{c}{\rho}=e \frac{c B}{p}
$$

defining the region of validity of L-DEM.

When this radiated power is of the same order of magnitude as the Lorentz power, then the particle energies stop growing and there is an upper limit of the frequency of the radiated photons, below the $\mathrm{TeV}$ range. This is model independent, since it is only related to L-DEM of individual particles, and it is in good agreement with the observed pulsar cut-off. If $\hat{P}=P_{\text {Dir }} / P_{\text {Lor }}$ and we consider $v \sim c$ and $\boldsymbol{E} \cdot \boldsymbol{v} \sim E c$, then

$$
\gamma \approx\left(\frac{\hat{P}}{\Omega \chi F}\right)^{1 / 3}
$$

and this combined with (3) gives

$$
\omega_{\text {peak }} \sim \frac{\hat{P}}{\chi F} \operatorname{rad~s}^{-1}
$$

with

$$
\begin{aligned}
F & =\frac{\left|\boldsymbol{E}_{\perp}+\boldsymbol{v} \times \boldsymbol{B}\right|}{E} \\
& =\left(\frac{m \Omega}{e B} \sqrt[3]{\frac{\hat{P}}{\Omega \chi}}\right)^{3 / 4} \text { when } \Omega \text { is constant }
\end{aligned}
$$

Thus for the Crab pulsar (CP), assuming that each particle keeps close to the same rotating magnetic field 
(MF) line, and assuming that we are close to the speed of light cylinder (SLC), the radii of curvature of the trajectories are close to the SLC radius, and therefore $\Omega \sim 200$ rad s${ }^{-1}$, the angular velocity of the CP. If $\hat{P} \in\left[10^{-4}, 1\right]$, $F \in\left[10^{-4}, 10^{-3}\right]$, and $\omega_{\max } \in\left[7 \times 10^{24}, 7 \times 10^{25}\right] \mathrm{rad} \mathrm{s}^{-1}$, and the maximum value of $\hbar \omega_{\max }<50 \mathrm{GeV}$.

A similar conclusion might be found using a simplified 2-dimensional perpendicular model of the pulsar, as may be see in da Costa [29] for flows close to the SLC. After a sharp growth in energy and frequency of radiated power, $\omega_{\text {peak }}$ reaches a value which is close to the value predicted theoretically. The difference is a result of the value of the radius of curvature of the trajectory, which is not exactly the value of the SLC radius, as we assumed, but very close to it. After growing steeply, $\hbar \omega_{\text {peak }}$ continues growing but in length scales which suggests it will never reach $\mathrm{TeV}$ values. This agrees with the observational cut-off $[1,2]$.

However the L-DEM is not valid everywhere in the magnetosphere. Two previous papers ([23, 30], Paper I and II respectively), pointed out that in the interval where L-DEM has to be used, the radiation is not a continuous process, but a discrete (random) one, because the peak radiation frequency is in the hard gamma-ray domain. This implies that the single particle motion is strongly influenced by this randomness. Therefore the motion calculation has to take it into account, translated into the combined effect of the stochastic nature of the distance between the radiation of two successive photons, and the random nature of the frequency of the radiated photons. Moreover the continuous description is not consistent with the average time and length scales of photon radiation. As seen in Paper II, in the case of the Crab pulsar, the charged particle mean free time between the radiation of two successive photons and the relaxation time for a particle to radiate its energy are larger than the equivalent classical trajectory calculations.

The radiative discrete (random) processes imply that trajectory calculations have to be done using Lorentz force, discounting at each random radiating point the random momentum of the photon radiated, recalculating the energy and restarting the calculation. Now particles can gain energy beyond the one for the balance between $P_{\text {Dir }}$ and $P_{\text {Lor }}$, accounting for further relevant fundamental processes, e.g. the balance between $\hbar \omega_{\text {peak }}$ and $\gamma m c^{2}$ which for classical $\hbar \omega_{\text {peak }}$ can turn out to be now $\hbar \omega_{\text {peak }}>\gamma m c^{2}$, an impossibility, since radiated photons cannot have energies greater than the particles which radiate them, and therefore $\hbar \omega_{\text {peak }}<\gamma m c^{2}$ always. This supersedes the calculation done by Higgins \& Henriksen $[6,7]$, and to achieve this we have to go beyond the classical radiation theory of high energy electrons.

In fact the classical general power spectrum of radiated photons, $P_{r}(\boldsymbol{r}, \boldsymbol{p}, \omega, t)$ is given by (4). Of course the radiation happens in a cone centered with axis parallel to $\boldsymbol{p}$ and aperture $\gamma^{-1}$, and therefore the radiation is collimated in a very narrow beam, and this phenomenon may be treated as occurring nearly aligned to $\boldsymbol{p}$. The power spectrum of radiation $P_{r}(\boldsymbol{r}, \boldsymbol{p}, \omega, t)$ can be defined, after integration over all directions $\boldsymbol{n}$ inside the cone, as

$$
P_{r}(\boldsymbol{r}, \boldsymbol{p}, \omega, t)=\int \mathrm{d} \boldsymbol{n} P_{r n}(\boldsymbol{r}, \boldsymbol{p}, \omega, \boldsymbol{n}, t)
$$

which combined with (6) is a condition of consistency to use the L-DEM. This reinforces the principle that the classical description of motion formally permits the spectral range of $P_{r}(\boldsymbol{r}, \boldsymbol{p}, \omega, t)$ to be $0 \rightarrow \infty$, with, of course,

$$
\left[P_{r}(\boldsymbol{r}, \boldsymbol{p}, \omega>c p / \hbar, t)\right] \neq 0
$$

violating the principle of conservation of energy, since allows the radiation of photons more energetic than the particles which radiate them. Classically this does not create any problem, provided the classical value $\hbar \omega_{c} \ll$ $\gamma m c^{2}$, and then

$$
\int_{0}^{c p / \hbar} d \omega P_{r}(\boldsymbol{r}, \boldsymbol{p}, \omega, t) \gg \int_{c p / \hbar}^{\infty} d \omega P_{r}(\boldsymbol{r}, \boldsymbol{p}, \omega, t)
$$

already not a fully correct formulation. However for $\gamma>\sqrt{2 m c^{2} /(3 \hbar \Omega)},\left(\hbar \omega_{c}>c p\right)$, the inequality (17) with classical $P_{r}(\boldsymbol{r}, \boldsymbol{p}, \omega, t)$ is no longer valid and does not make sense at all. Moreover to correct these unphysical relation (16) must change into

$$
\begin{array}{r}
P_{r}(\boldsymbol{r}, \boldsymbol{p}, \omega<c p / \hbar, t) \neq 0 \\
\lim _{\omega \rightarrow c p / \hbar} P_{r}(\boldsymbol{r}, \boldsymbol{p}, \omega<c p / \hbar, t)=0 \\
P_{r}(\boldsymbol{r}, \boldsymbol{p}, \omega \geq c p / \hbar, t)=0 .
\end{array}
$$

and the value of $\hbar \omega_{c}$ has to be changed

$$
1.5 \hbar \Omega \gamma^{3} \rightarrow \alpha \gamma m c^{2} \quad(\alpha \in[0,1]),
$$

and this can only be achieved introducing a quantum description to $P_{r}(\boldsymbol{r}, \boldsymbol{p}, \omega, t)$, which will contain the classical description. Then $P_{\text {Dir }}$ loses completely its physical meaning, as it is no longer $P_{\text {Rad }}$, and this is aggravated by the vacuum polarization problem [31] and its associated curve-Cerenkov radiation.

To fully model these mechanisms it is essential to go beyond the single particle approach, thus avoiding limitations it incurs due to the randomness of the associated radiative process. Instead we must model the plasma flow in the magnetosphere, requiring a study of the collective behaviour of the plasma in order to understand the variability of such high energetic photons, which is also the motivation for the study of the plasma flow in pulsar magnetospheres. However this requires a relativistic kinetic theory of radiative plasmas: in other words, an extension of the general theory of collisionless plasmas when no radiative effects are present that is the current basis of the modeling of the plasma flow in the magnetospheres. Since the theory of collisionless non-radiative plasmas is the Vlasov theory with its formal equivalence with standard orbit theory [17], we require that the kinetic theory of radiative plasmas has a formal equivalence 
with a generalized orbit theory defined rigorously in the presence of stochastic radiative effects. The application of such theory only makes sense if the particle density is high enough to smooth the random effects introduced by the radiative process, although it seems that the random effects are amplified in the collective approach (Paper II and [21]). More detailed microstability analysis will be the subject of further study.

To fulfill this goal this paper is divided in two further sections. We introduce the concept of the quasiclassical (q-classical) and quasi-quantum (q-quantum) plasma flows in section II, going beyond the classical description, defining the orbital motion of individual charged particles in the general case where the momentum radiated has a significant effect on the onward trajectory of the radiator, and defines the generalized orbit theory, showing at the same time why the analysis cannot be done using just a single particle approach. The physics is further developed over five subsections: II.A concentrates on the complete theory of curvature radiation, with its q-classical and q-quantum approach; II.B generalises the two previous subsections to address collective phenomena and the formulation of the kinetic theory of the relativistic plasmas; II.C specialises the preceding analysis to the particular case of the rotating pulsar; finally, II.D introduces a further complication in the radiation losses by considering the implications of vacuum polarisation, and the possibility of Cerenkov radiation in vacuum. A concluding discussion follows in the final section, along with a description of future work.

Three appendices are provided, for the reader's convenience: the first gives a list of variables used in the paper, along with their definitions; the second establishes the intimate relationship between synchrotron and curvature radiation; the third gives mathematical detail which is removed from the main text in the interests of clarity.

In the treatment given in this paper, we conclude that the radiative limit is given through the calculation of the maximum value of $\omega$ allowed by $\hbar \omega \rightarrow c p$, when accounting for the discreteness and extreme energy of the radiation, and not in terms of balancing $P_{\text {Rad }}$ with $P_{\text {Lor }}$. Then we recover this balance, without any direct role in dynamical calculations, as a consequence of the law of large numbers, where there is indeed a transition point at around $50 \mathrm{GeV}$, as in the classical case, but this is not a fixed cut-off: indeed, particles can continue to radiate far beyond this energy, up to almost the total energy of the particle at $\gamma \sim 10^{9}$ - a point at which the classical model is useless. This brings a further bonus, the discrete model allows photons of $100 \mathrm{~s}$ of $\mathrm{TeV}$ to be produced in a self-consistent physical way, with the radiating particles losing almost all of their energy in such production.

Therefore we will get as a conclusion that we have a real cut-off at $\mathrm{TeV}$ energies beyond which no radiative processes are possible, superseding the observed classical cut-off at GeV range, in fact an abrupt fall of the gammaray radiated power with frequency.

\section{THE QUASI-CLASSICAL PLASMA FLOW}

Generalized orbit theory as required by the new kinetic theory of radiative plasmas, contains the standard orbit theory, when no radiative processes are considered. Classical trajectories only have to be redefined when the radiative process starts to be important. This happens for a value of $p_{q c l}$ given as follows.

The probability distribution $P_{p}(\boldsymbol{r}, \boldsymbol{p}, \omega, t)$ of radiating a photon at radiation point is given by

$$
P_{p}(\boldsymbol{r}, \boldsymbol{p}, \omega, t)=(\hbar \omega)^{-1} P_{r}(\boldsymbol{r}, \boldsymbol{p}, \omega, t) .
$$

The average number of photons radiated by unit time is

$$
\frac{d\left\langle N_{p h}\right\rangle}{d t}=\lambda(\boldsymbol{r}, \boldsymbol{p}, t)=\int_{0}^{\gamma m c^{2} / \hbar} \mathrm{d} \omega P_{p}(\boldsymbol{r}, \boldsymbol{p}, \omega, t)
$$

However we may consider a value of $\omega_{c}<\omega_{\max }<c p / \hbar$ such that

$$
\lambda(\boldsymbol{r}, \boldsymbol{p}, t) \approx \int_{0}^{\omega_{\max }} \mathrm{d} \omega P_{p}(\boldsymbol{r}, \boldsymbol{p}, \omega, t)
$$

with

$$
\int_{\omega_{\max }}^{\gamma m c^{2} / \hbar} \mathrm{d} \omega P_{p}(\boldsymbol{r}, \boldsymbol{p}, \omega, t) \ll \int_{0}^{\gamma m c^{2} / \hbar} \mathrm{d} \omega P_{p}(\boldsymbol{r}, \boldsymbol{p}, \omega, t)
$$

This is a consequence of a set of values of

$$
\omega>\omega_{c}: P_{p}(\boldsymbol{r}, \boldsymbol{p}, \omega, t) \ll P_{p}\left(\boldsymbol{r}, \boldsymbol{p}, \omega_{\text {peak }}, t\right)
$$

the upper stream tail of the distribution, and this means that there are very few photons radiated for $\omega>\omega_{\max }$.

Then

$$
\begin{gathered}
{\left[p_{q c l}: \hbar \omega_{\max }\left(p_{q c l}\right) \sim m c^{2}\right]} \\
\Rightarrow\left[p>p_{q c l}: \hbar \omega_{\max }(p)>m c^{2}\right] .
\end{gathered}
$$

which defines the classical $\Longleftrightarrow$ q-classical boundary, as the radiation can still be described classically.

In an individual particle motion, the radiative process occurs at well determined discrete random events clearly separated in time and space, and therefore it is still possible to assume charged particle trajectories between them, created by the action of Lorentz force as seen in Paper II, whose description we shall follow in this section.

For particles with momentum $p>p_{q c l}$ the stochastic radiative process is defined by quantum curvature radiation theory, which may be simplified to the classical one, for $\hbar \omega_{c} \ll c p$. This defines two descriptions of motion based on classical trajectories driven by Lorentz force: either the q-classical description when the radiation power spectrum can be defined classically, or the q-quantum one when a full quantum description is required.

As the photons are radiated at random times, directions and random frequencies, this is a compound Poisson process, particular case of a marked point process 
$[23,32]$. Thus the overall trajectories are not deterministic but stochastic, because there is randomness in the choice of trajectories after radiation, depending on the stochastic nature of the energy and direction of the radiated photons. Nevertheless the trajectories remain collimated, provided the radiated energies are similar to the classical calculation.

In this marked point process, the probability of radiating a number of photons $N_{p h}$ in the interval $(t-\Delta t / 2, t+$ $\Delta t / 2)$ is the generalized Poisson distribution ([30]) where $\lambda(\boldsymbol{r}, \boldsymbol{p}, t)$ is not only the probability of radiating a photon per unit time but also the average number of photons per unit time.

There are two probability distributions of two random variables connected by the parameter $\lambda(\boldsymbol{r}, \boldsymbol{p}, t)$ : the interval of time between the radiation of two successive photons has probability distribution [23]

$$
f_{t}(\tau)=\lambda(\boldsymbol{r}, \boldsymbol{p}, t+\tau) \exp \left[-\int_{t}^{t+\tau} \mathrm{d} t \lambda(\boldsymbol{r}, \boldsymbol{p}, t)\right]
$$

allowing the calculation of a mean free path and a mean free time of radiation; and the frequency of radiation at radiative points already defined.

This allows the definition of a new relevant stochastic parameter

$$
\xi=\frac{e \boldsymbol{E} \cdot \boldsymbol{v} \delta t}{\hbar \omega}=\frac{P_{\text {Lor }}}{P_{\text {Rad }}} \frac{\beta_{1}}{\beta_{2}}
$$

where $\delta t=\beta_{1} / \lambda(\boldsymbol{r}, \boldsymbol{p}, t)$ and $\hbar \omega=\beta_{2} \hbar\langle\omega\rangle>m c^{2}$, being $\beta_{1}$ and $\beta_{2}$ stochastic variables, calculated through Eqs. $(26,20)$ respectively, and $\langle\omega\rangle$ is associated with the real meaning of

$$
\begin{aligned}
P_{\text {Rad }}(\boldsymbol{r}, \boldsymbol{p}, t) & =\int_{0}^{\gamma m c^{2} / \hbar} \mathrm{d} \omega P_{r}(\boldsymbol{r}, \boldsymbol{p}, \omega, t) \\
& =\int_{0}^{\gamma m c^{2} / \hbar} \mathrm{d} \omega \hbar \omega P_{p}(\boldsymbol{r}, \boldsymbol{p}, \omega, t) \\
& =\hbar \lambda(\boldsymbol{r}, \boldsymbol{p}, t)\langle\omega\rangle
\end{aligned}
$$

as it is the average photon energy, multiplied by the number of average photons radiated per unit time, as seen in Paper II. In the definition of $\xi$ we are putting $P_{\text {Rad }}$ instead of $P_{\text {Dir }}$, because as seen, in a quantum context, $P_{\text {Dir }}$ has no physical meaning. $\xi<1$ means that the particle is losing energy; $\xi>1$ means that the particle is gaining energy. This parameter shows that although we may have $P_{\text {Lor }} / P_{\text {Rad }}<1$, we may have $\xi>1$. The problem is even more severe in extreme radiative conditions.

When $\xi \gg 1$ no radiative corrections are required as in the classical case. As found in Paper II this also happens after crossing the classical $\Longleftrightarrow$ q-classical boundary: although there are radiation points, they may be ignored when the correspondent radiated photons $\hbar \omega \lesssim m c^{2}$.

In operational terms the classical domain may be extended: following (25), we may choose a value $\omega_{\mathrm{b}}$ such that

$$
\omega_{b}=\omega_{\max }(p)=\chi \omega_{c}=\kappa c p / \hbar
$$

where $\kappa$ and $\chi$ are chosen to satisfy equation (23). Then following inequality $(24)[26,28]$, as shown in Figure 1

$$
5<\chi \ll \gamma^{-2} \frac{m c^{2}}{\hbar \Omega}
$$

this implies

$$
\begin{aligned}
\gamma_{b} & =\sqrt{\frac{2 \kappa}{3 \chi} \frac{m c^{2}}{\hbar \Omega}} \\
\omega_{b} & =\Omega \sqrt{\frac{2}{3 \chi}}\left(\frac{m c^{2}}{\hbar \Omega} \kappa\right)^{3 / 2}
\end{aligned}
$$

meaning that photons with energies $\hbar \omega<\hbar \omega_{b}$ do not change appreciably the particle's energy and momentum.

Since $\omega_{\max }=\omega_{\max }(\boldsymbol{p})$ and $\omega_{\mathrm{b}}=$ const, we only get significant radiative influence on the motion of charged particles for photons when $\omega_{\max }>\omega_{\mathrm{b}}$, and therefore when $\gamma>\gamma_{\mathrm{b}}$, and therefore the number of particles which radiate significantly per unit time is

$$
\frac{d\left\langle N_{p h}^{\prime}\right\rangle}{d t}=\lambda^{\prime}(\boldsymbol{r}, \boldsymbol{p}, t)=\int_{\omega_{b}}^{\omega_{Q}} \mathrm{~d} \omega P_{p}(\boldsymbol{r}, \boldsymbol{p}, \omega, t)
$$

with $\omega_{Q}=\max \left(\omega_{\mathrm{b}}, \omega_{\max }\right)$ redefining the mean free path and mean free time of radiation. This redefines the extension of the classical domain for operational reasons, as then $\omega_{\max }(p)<\omega_{\mathrm{b}}$, implying $\lambda^{\prime}(\boldsymbol{r}, \boldsymbol{p}, t)=0$, and consequently equivalent infinite mean free time and mean free path of radiation.

The random nature of the value of $\xi$, with different values for each particle, makes it difficult to characterize the evolution of the electron-positron plasma acted upon by Lorentz force, in a single particle approach context. The use of a collective approach is therefore compulsory, but it requires the radiation power spectrum covering both the q-classical and q-quantum regimes. Therefore in A below, the single-particle radiation term is revisited using the correct relativistic expressions for the photon spectrum; sub-section B goes on to define the strategy for incorporating the new 'collision' term (that is, the correct radiation recoil) in the distribution function description.

\section{A. Curvature Radiation}

Let us define $\Upsilon$ the ratio of the "classical" $\hbar \omega_{c}$ over $\varepsilon=\gamma m c^{2}$, the particle energy. Then

$$
\begin{aligned}
\Upsilon & =\frac{3 \hbar}{2 \varepsilon} \frac{c}{\rho} \gamma^{3} \\
& =\frac{3 \hbar \Omega}{2 m c^{2}} \gamma^{2} \text { for } \Omega \text { fixed } \\
& =\frac{3 \hbar e\left|\boldsymbol{E}_{\perp}+\boldsymbol{v} \times \boldsymbol{B}\right|}{2 m^{2} c^{3}} \gamma
\end{aligned}
$$

where we made the transposition $B \Rightarrow\left|\boldsymbol{E}_{\perp}+\boldsymbol{v} \times \boldsymbol{B}\right| / c$ from synchrotron radiation (Appendix $\mathrm{B}$ ), and $c / \rho$ is 
given by Eq. (9). As we have seen in Section I, we may have $\Upsilon \gg 1$, and therefore we move outside the classical domain into the quantum domain. Thus we need to consider the physics of curvature radiation in both the q-classical and q-quantum regimes, to provide an overall definition of $P_{r}(\boldsymbol{r}, \boldsymbol{p}, \omega, t)$. Provided $\gamma \gg 1$ [33] and following Berestetskii et al [34], the particle quantum radiative power spectrum is for all $\Upsilon$ :

$$
\begin{aligned}
P_{r}(\boldsymbol{r}, \boldsymbol{p}, \omega, t) & =\frac{3^{5 / 2}}{8 \pi}(1+\Upsilon)^{-2} P_{\operatorname{Dir}} \frac{\omega}{\omega_{c}^{2}}\left[\int_{\zeta \frac{\omega}{\omega_{c}}}^{\infty} K_{5 / 3}(\eta) \mathrm{d} \eta-\right. \\
& \left.-(3 \pi)^{-1 / 2} \frac{\Upsilon^{2}}{1+\Upsilon}\left(\frac{\omega}{\omega_{c}}\right)^{2} \zeta K_{2 / 3}\left(\zeta \frac{\omega}{\omega_{c}}\right)\right]
\end{aligned}
$$

with

$$
\begin{aligned}
\zeta(\Upsilon, \omega) & =\frac{1}{1+\Upsilon} \frac{1}{1-(\hbar \omega / \varepsilon)} \\
& =\left[1+\Upsilon\left(1-\frac{\omega}{\omega_{c}}\right)\right]^{-1} \\
\hbar \omega_{c} & =\frac{\varepsilon \Upsilon}{1+\Upsilon}
\end{aligned}
$$

where $P_{\text {Dir }}$ is the classical radiation constant from before (Eq. (5)). As $\hbar \omega \lesssim \varepsilon$, since the particle cannot radiate photons more energetic than the particles themselves, then

$$
\frac{\omega}{\omega_{c}} \in[0,1+1 / \Upsilon)
$$

and the conditions of Eqns. $(18,19)$ are fully met.

Eqn. (37) is similar to Eqn. (4) for the classical and the q-classical domains, where the second term is negligible compared with the integral of $K_{5 / 3}(\eta)$, when

$$
\begin{aligned}
\Upsilon \ll 1 & \Longrightarrow \omega_{c}=1.5 \Omega \gamma^{3} \quad \text { (and from Eqn. (8)) } \\
& \Longrightarrow \hbar \omega_{\text {peak }} \sim 0.29 \hbar \omega_{c} \ll \gamma m c^{2} \\
& \Longrightarrow \hbar \omega_{\max } \sim \chi \hbar \omega_{c} \ll \gamma m c^{2}
\end{aligned}
$$

being $\omega_{\max }$ as defined by relation (23), with $\chi$ obeying relation (30). Then Eqn. (38) for $\hbar \omega \ll \gamma m c^{2}$ contains Schwinger [27] correction, and $\omega_{\max } / \omega_{c} \sim 1 / \Upsilon \gg 1$ consistent with the classical approximation $1 / \Upsilon \Rightarrow \infty$.

However when either $\gamma$ or the EM field grows, then $\Upsilon$ grows as well, and we move from the q-classical into the q-quantum domain. According to Berestetskii et al [34], for $\Upsilon \gg 1, P_{r}(\boldsymbol{r}, \boldsymbol{p}, \omega, t)$ grows very little with frequency in the interval $\omega / \omega_{c} \in(0.5,0.8)$, dropping afterwards to zero as $\omega / \omega_{c} \Rightarrow 1+1 / \Upsilon$, as can be seen in Figure 1 and therefore

$$
\begin{aligned}
\Upsilon \gg 1 & \Longrightarrow \hbar \omega_{c} \simeq(1-1 / \Upsilon) \gamma m c^{2} \\
& \Longrightarrow \hbar \omega_{\text {peak }} \sim 0.8 \hbar \omega_{c} \sim 0.8 \gamma m c^{2} \\
& \Longrightarrow \hbar \omega_{\max } \sim \hbar \omega_{c} \sim \gamma m c^{2}
\end{aligned}
$$

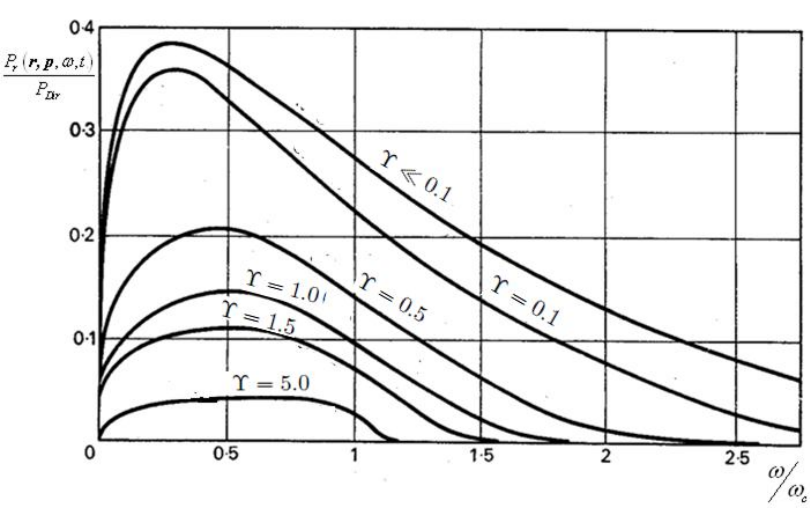

FIG. 1: This figure, taken from Berestetskii et al [34], shows the representation of $P_{r}(\boldsymbol{r}, \boldsymbol{p}, \omega, t) / P_{\text {Dir }}$ for different values of $\Upsilon$. When $\Upsilon \ll 0.1$ the radiating electrons are in the classical and q-classical regime. The q-quantum regime will happen for $\Upsilon \gg 1$, and therefore $\Upsilon \sim 5$ gives a good picture of the radiative spectrum for q-quantum regimes. There the peak frequency of radiation is not as sharply defined as for the $\Upsilon \ll 0.1$ case, but is around $0.8 \omega_{c}$, implying $\hbar \omega_{\max } \sim \gamma m c^{2}$.

\section{Close to star surface}

With these expressions we may show that for the surface of the pulsar the associated $\omega$ and $\gamma \sim O(1)$, and therefore we are in a quantum domain where expression (37) can not be used.

In fact assuming $B_{s} \sim 10^{8} \mathrm{~T}$, then $\Upsilon=3.47 \times 10^{-2} \gamma$, for $\omega=\omega_{c}$ the equation relating both quantities is

$$
\hbar \omega=\frac{3.47 \times 10^{-2} \gamma^{2}}{1+3.47 \times 10^{-2} \gamma} m c^{2}
$$

If we put $\hbar \omega=\alpha m c^{2}$ then the solution is

$$
\gamma=\frac{\alpha+\sqrt{\alpha^{2}+115.76 \alpha}}{2}
$$

The boundary between classical $\Longleftrightarrow$ q-classical domains is when $\alpha=1$, and then $\gamma \sim 6 \sim \mathcal{O}(1)$. The conclusion is that no such boundary exists for EM Fields close to the critical value $B_{C L} \sim 4.47 \times 10^{9} \mathrm{~T}$, since we are dealing with a quantum plasma which requires quantum treatment [33]. But when $\alpha \sim 10^{4}$ corresponding to a photon energy of $5 \mathrm{GeV}$, then $\gamma \sim 1.0003 \times 10^{4}$, allowing the use of Eq. (37) giving a $\Upsilon \sim 2360$. This latter value of $5 \mathrm{GeV}$ is indeed the upper value of radiated photon energies, not very useful for the theory of pulsar cut-off, but excellent for pair production, since here [34]

$$
P_{\mathrm{Rad}}(\boldsymbol{p}, t)=\eta[\Upsilon(\gamma)] P_{\mathrm{Dir}}(\boldsymbol{p}, t)=\int_{0}^{c p / \hbar} \mathrm{d} \omega P_{r}(\boldsymbol{r}, \boldsymbol{p}, \omega, t)
$$

where

$$
\eta[\Upsilon(\gamma)]= \begin{cases}1, & \Upsilon \ll 1(\text { classical regime }) \\ <1, & \text { otherwise }\end{cases}
$$


and this fulfils the condition $P_{L o r} \sim P_{\text {Rad }}$ since

$$
\gamma \sim\left(\frac{m c}{\eta[\Upsilon(\gamma)] e \chi\left|\boldsymbol{E}_{\perp}+\boldsymbol{v} \times \boldsymbol{B}\right| F}\right)^{1 / 2}
$$

with $\eta(24) \sim 0.01$, and the radiated photons have energies smaller than the particles which radiate them. We can see that $5 \mathrm{GeV}$ photons can be radiated by particles with $\gamma \sim 10^{4}$, much less than the $\gamma \sim 10^{7}$ required in the classical case, and these photons can be generated close to the stellar surface.

Equation (50) is the same for the classical case where $\eta[\Upsilon(\gamma)]=1$. But it cannot be used in this context because now $\Upsilon \gg 1$, and therefore the "classical" photons have energies which are more energetic than the particle themselves.

\section{Close to the speed of light cylinder}

Revisiting the calculations in Paper II for particles moving close to the SLC, and $\Omega=200 \mathrm{rad} \mathrm{s}^{-1}$ and putting $\Upsilon(\gamma)$ with $\Omega$ constant, then $\Upsilon\left(10^{7}\right)=2.5 \times$ $10^{-5} \ll 1, \Upsilon\left(10^{8}\right)=2.5 \times 10^{-3} \ll 1, \Upsilon\left(10^{9}\right)=0.25$, and $\Upsilon\left(10^{10}\right)=25$. Therefore a particle which is allowed to reach high values of $\gamma$ will lose most of its energy in a single radiation, returning to the classical radiative domain or close to it.

However close to SLC a particle does not require its Lorentz factor to go much beyond $\gamma \sim 10^{8}$ to radiate with $\Upsilon \gg 1$. If the magnitude of the EM field increases suddenly one or two orders of magnitude, the radius of curvature becomes smaller, and therefore the value of $\Upsilon$ jumps, creating the conditions for the particles to radiate photons with energies of the order of the particles themselves, and the particle loses momentum or even stops. This can be concluded in a fully consistent picture for the collective behaviour of the whole plasma, addressed in the next subsection.

\section{B. The collective phenomena and evolutionary process}

We are going to assume that the electron-positron collisionless plasma starts to flow from rest, in an EM field, and therefore is accelerated by the Lorentz force. Where the average energy of the plasma is relatively low $\left(p<p_{b}\right)$, we shall disregard the influence of radiation on the trajectories of individual particles: we are in the classical domain, where radiation is given by classical curvature radiation theory, with $\Upsilon \ll 1$ and $\gamma \gg 1$, but where the radiated photons do not influence the plasma motion. However, for those particles in the distribution with momentum exceeding the threshold $p_{\mathrm{b}}$, their evolution must take into account radiation recoil where it is significant.

We shall defer a further complication until a later publication: the seeding of the distribution function by pair production arising directly from the decay of high-energy photons. Hence we have 'plasma recycling': the process in which the most energetic particles radiate very strongly, losing most of their energy, and are then reaccelerated by the ambient fields to energies where energetic radiation is again possible.

The kinetic equation in the classical regime is the usual Vlasov equation. The plasma will cross the boundary defined by condition (25) and will enter the q-classical region, where radiative effects need to be taken into account, and the Vlasov description is extended by incorporating a radiation term (see Appendix $\mathrm{C}$ ). This assumption excludes the case where due to the extreme strength of the EM fields, the region for $\gamma>1$ is already a quantum region.

Taking account the presence of electrons (-) and positrons $(+)$, we have to introduce two distribution functions, one for each species. In the extended classical domain, when no radiative effects need to be taken into account, we can define the effective minimum and maximum of the momentum distribution $p_{\min }, p_{\max }$ such that $p_{\max }<p_{\mathrm{b}}$, and

$$
f_{ \pm}(\boldsymbol{r}, \boldsymbol{p}, t)=\left\{\begin{array}{l}
\neq 0, \text { for } p_{\min }<p<p_{\max } \\
\sim 0, \text { otherwise }
\end{array}\right.
$$

The general kinetic equations for both distributions, with the previous assumptions and $\left(\delta f_{ \pm} / \delta t\right)_{S E}$ the change in the distribution functions due to spontaneous emission of photons, will be

$$
\begin{aligned}
\frac{D f_{ \pm}}{D t} \equiv & \frac{\partial f_{ \pm}}{\partial t}+\boldsymbol{v} \cdot \nabla f_{ \pm} \\
& +\left[\boldsymbol{E}\left(f_{ \pm}\right)+\boldsymbol{v} \times \boldsymbol{B}\left(f_{ \pm}\right)\right] \cdot \nabla_{p} f_{ \pm} \\
= & \left(\frac{\delta f_{ \pm}}{\delta t}\right)_{S E}
\end{aligned}
$$

where when $f_{ \pm}(\boldsymbol{r}, \boldsymbol{p}, t)$ is in the classical region

$$
\left(\frac{\delta f_{ \pm}}{\delta t}\right)_{S E}=0
$$

and when $f_{ \pm}(\boldsymbol{r}, \boldsymbol{p}, t)$ is inside the q-classical and qquantum regions

$$
\left(\frac{\delta f_{ \pm}}{\delta t}\right)_{S E}=\mathcal{N}_{c}(\boldsymbol{r}, \boldsymbol{p}, t)-\lambda(\boldsymbol{r}, \boldsymbol{p}, t) f_{ \pm}(\boldsymbol{r}, \boldsymbol{p}, t)
$$

where

$$
\begin{aligned}
\mathcal{N}_{c}(\boldsymbol{r}, \boldsymbol{p}, t)=\int \mathrm{d} \boldsymbol{n} \int_{0}^{\omega_{\mathrm{pm}}} d \omega P_{p n}\left(\boldsymbol{r}, \boldsymbol{p}+\frac{\hbar \omega}{c} \boldsymbol{n}, \omega, \boldsymbol{n}, t\right) \\
\times f_{ \pm}\left(\boldsymbol{r}, \boldsymbol{p}+\frac{\hbar \omega}{c} \boldsymbol{n}, t\right)
\end{aligned}
$$

with

$$
P_{p n}(\boldsymbol{r}, \boldsymbol{p}, \omega, \boldsymbol{n}, t)=\frac{P_{r n}(\boldsymbol{r}, \boldsymbol{p}, \omega, \boldsymbol{n}, t)}{\hbar \omega}
$$


and $\omega_{\mathrm{pm}}: \boldsymbol{c p}+\hbar \omega_{\mathrm{pm}}\left(p_{\max }\right) \boldsymbol{n}=c \boldsymbol{p}_{\max }$. In the above equations everything happens in fixed position in configuration space $(\boldsymbol{r}, \boldsymbol{p})$. In equation (54), the first term, $\mathcal{N}_{c}(\boldsymbol{r}, \boldsymbol{p}, t)$, gives the density of particles per unit time which have been scattered into $\boldsymbol{p}$ from a higher momentum by photon radiation; the second term gives the density of particles per unit time which are lost to $\boldsymbol{p}$ by radiation.

Since for each $\boldsymbol{p}$ the range of radiated frequencies is $\omega \in\left[\omega_{\mathrm{b}}, \omega_{\max }(p)\right]$, eq, (54) may be rewritten into

$$
\begin{aligned}
\left(\frac{\delta f_{ \pm}}{\delta t}\right)_{S E}=\int \mathrm{d} \boldsymbol{n} & \int_{\omega}^{\omega_{\mathrm{cal}}} d \omega P_{p n}\left(\boldsymbol{r}, \boldsymbol{p}+\frac{\hbar \omega}{c} \boldsymbol{n}, \omega, \boldsymbol{n}, t\right) \\
& \times f_{ \pm}\left(\boldsymbol{r}, \boldsymbol{p}+\frac{\hbar \omega}{c} \boldsymbol{n}, t\right) \\
& -\lambda^{\prime}(\boldsymbol{r}, \boldsymbol{p}, t) f_{ \pm}(\boldsymbol{r}, \boldsymbol{p}, t)
\end{aligned}
$$

where $\omega_{\text {cal }}=\max \left\{\omega_{\mathrm{b}}, \min \left[\omega_{\mathrm{pm}}\left(p_{\max }\right), \omega_{\max }\left(p_{\max }\right)\right]\right\}$. When $\min \left[\omega_{l}\left(p_{\max }\right), \omega_{\max }\left(p_{\max }\right)\right]<\omega_{\mathrm{b}}$, eq. (57) reduces to eq. (53) we are in the extended classical domain, and the kinetic equation is the standard Vlasov equation.

The distribution functions $f_{ \pm}(\boldsymbol{r}, \boldsymbol{p}, t)$ governed by Eqs. $(52,54)$ act as sources for the self-electromagnetic fields:

$$
\begin{gathered}
\nabla \cdot \boldsymbol{E}_{f}=\frac{e}{\epsilon_{0}} \int_{\text {all } \boldsymbol{p}} \mathrm{d} \boldsymbol{p}\left[f_{+}(\boldsymbol{r}, \boldsymbol{p}, t)-f_{-}(\boldsymbol{r}, \boldsymbol{p}, t)\right] \\
\nabla \times \boldsymbol{B}_{f}=e \mu_{0} \int_{\text {all } \boldsymbol{p}} \mathrm{d} \boldsymbol{p} \frac{c \boldsymbol{p}}{\sqrt{m^{2} c^{2}+p^{2}}}\left[f_{+}(\boldsymbol{r}, \boldsymbol{p}, t)-\right. \\
\left.-f_{-}(\boldsymbol{r}, \boldsymbol{p}, t)\right]+\frac{1}{c^{2}} \frac{\partial \boldsymbol{E}_{f}}{\partial t}
\end{gathered}
$$

and they are related by Faraday's Law.

Now the formal equivalence between the Vlasov equation and its extension (see Appendix C) with orbit theory may be used [17]. Consider the distribution function of momentum at a snapshot in time, but at different positions along the accelerating fields; equivalently, we may think of the evolution of the distribution function convected from an initial position at the appropriate mean speed, over a range of times (with the caveat that selffield fluctuations are also evolving). At early positions (or times) the distribution function in momentum space has a particular shape, with virtually no particles with zero momentum (because the plasma is under continuous acceleration). Evolution from this point means that an increasing fraction of the distribution function tail exceeds the threshold at which energetic radiation consequences must be considered, and we see two effects: the virtual truncation of the high-momentum tail as radiation effects cause significant momentum loss, and the simultaneous evolution of a low-momentum component arising directly from the recoil produced by such radiation. Figure 2 captures the essence of this behaviour, showing sketches of the distribution functions at each critical stage.

To understand how this happens let us consider the set of cells $(\Delta \boldsymbol{r}, \Delta \boldsymbol{p})$ centered at $(\boldsymbol{r}, \boldsymbol{p})$ at time $t$ in which

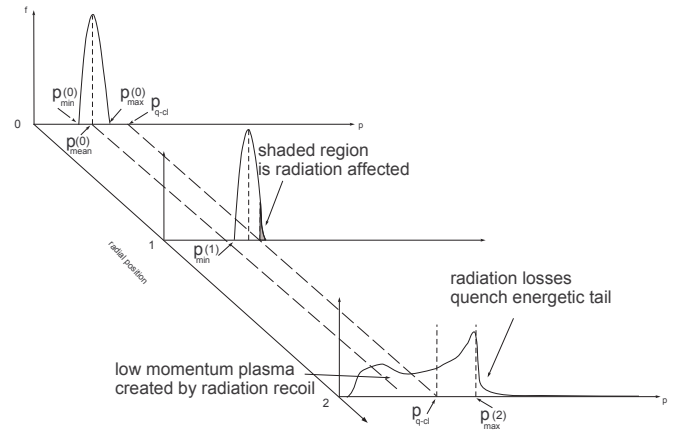

FIG. 2: Figure shows sketch of the distribution function (in momentum space) at different radial positions along the accelerating, at a snapshot in time, for a singe reference frame; it may also be considered as an evolutionary sketch for the distribution function as it develops in time. At early radial positions close to the star (that is, position 0 in the diagram), the distribution function of particle momenta has an arbitrary form with a non-zero minimum momentum, and a maximum that is below the threshold for which radiation recoil may become important. At position 1, the minimum momentum has grown (since the distribution is under continuous acceleration, and we are using the same reference frame as at position 0), and the maximum momentum is now sufficiently high that radiation effects need to be taken into account. At position 2 , the distribution function has been significantly altered by radiation recoil: there is an extended low-momentum (low energy) plasma at the same time as a quenching of the highenergy tail after $p_{\max }$. Note that these sketches are not to scale, and that the high energy tail (above $p_{q c l}$ ) in reality is significantly longer than the lower part.

we may divide the $f\left(\boldsymbol{r}, \boldsymbol{p}: p \in\left(p_{\min }, p_{\max }\right), t\right)$. In the classical domain,

$$
\begin{aligned}
& f\left[\boldsymbol{r}, \boldsymbol{p}: p \in\left(p_{\min }, p_{\max }\right), t\right] \rightarrow \\
& f\left[\boldsymbol{r}+\Delta \boldsymbol{r}, \boldsymbol{p}^{\prime}: p^{\prime} \in\left(p_{\min }+P_{\text {Lor }} \Delta t / c\right.\right. \\
& \left.\left.p_{\max }+P_{\text {Lor }} \Delta t / c\right), t+\Delta t\right]
\end{aligned}
$$

as no decay exists $\left[\lambda^{\prime}\left(\boldsymbol{r}, \boldsymbol{p}_{\max }, t\right)=0\right]$. The extremes of the distribution function grow, but the width $\left(p_{\min }, p_{\max }\right)$ does not change appreciably.

But when $p_{\max }>p_{\mathrm{b}}$ we enter the q-classical regime, and we have to consider Vlasov equation radiative extension for all range $0<p<p_{\max }$. Considering $p^{\prime}=$ $p+P_{\text {Lor }} d t / c$, then the set of particles with a given $\boldsymbol{p}_{\text {max }}$, move into the interval $\left[p_{\max }^{\prime}-\hbar \omega_{\max }\left(p_{\max }\right) / c, p_{\max }^{\prime}\right]$, and the set of particles with $\boldsymbol{p}_{\min }$ move either into $\boldsymbol{p}_{\min }^{\prime}$ if $p_{\min }<p_{\mathrm{b}}$ or the interval $\left[p_{\min }^{\prime}-\hbar \omega_{\max }\left(p_{\min }\right) / c, p_{\min }^{\prime}\right]$, if $p_{\min }>p_{b}$, and then the distribution function starts widening, because

$$
\begin{aligned}
p_{\max }-\min \left[p_{\max }-\hbar \omega_{\max }\left(p_{\max }\right) / c,\right. & \\
& \left.p_{\min }-\hbar \omega_{\max }\left(p_{\min }\right) / c, p_{\min }\right] \\
>\quad & p_{\max }-p_{\min }
\end{aligned}
$$

and therefore $p_{\text {min }} \rightarrow 0$ along a trajectory, for growing $t$. 
Thus inside the q-classical region the distribution function takes a different form:

$$
f_{ \pm}(\boldsymbol{r}, \boldsymbol{p}, t)=\left\{\begin{array}{l}
\neq 0, \text { for } p<p_{\max } \\
\sim 0, \text { otherwise }
\end{array}\right.
$$

showing that the value of $p \in\left(0, p_{\max }\right)$ has many more cells, and eq. (54) shows that we may consider $f(\boldsymbol{r}, \boldsymbol{p}, t)$ to be divided into two ranges in momentum space, about the point $p_{m} \in\left(0, p_{\max }\right)$, defined by

$$
\begin{aligned}
& \int d \boldsymbol{n} \int_{\omega_{b}}^{\omega_{\mathrm{cal}}} \mathrm{d} \omega P_{p n}\left(\boldsymbol{r}, \boldsymbol{p}_{m}+\frac{\hbar \omega}{c} \boldsymbol{n}, \omega, \boldsymbol{n}, t\right) \times \\
& \times f\left(\boldsymbol{r}, \boldsymbol{p}_{m}+\frac{\hbar \omega}{c} \boldsymbol{n}, t\right)-\lambda^{\prime}\left(\boldsymbol{r}, \boldsymbol{p}_{m}, t\right) f\left(\boldsymbol{r}, \boldsymbol{p}_{m}, t\right)=0(
\end{aligned}
$$

that is, the point in momentum space in which population losses are exactly balanced by gains. Despite the action of the Lorentz force, there is a momentum range in which $p<p_{m}$, where $(\delta f / \delta t)_{S E}>0$, and this shows that the density of particles per unit time which have been scattered into $\boldsymbol{p}$ from a higher momentum by photon radiation overcomes the exponential decay, and therefore when $t \rightarrow t+\Delta t$ the number of particles grows in a moving cell; and another one in which $p>p_{m}$ where $(\delta f / \delta t)_{S E}<0$, and exponential decay dominates.

Despite the dominance of radiation losses for $p>$ $p_{m}, p \leq p_{\max }, p_{\max }$ itself grows, because the parameter which controls radiative decay $\lambda^{\prime}\left(\boldsymbol{r}, \boldsymbol{p}: p \lesssim p_{\max }, t\right)$, starts by being

$\lambda^{\prime}\left(\boldsymbol{r}, \boldsymbol{p}: p=p_{b}, t\right)=0 \rightarrow \lambda(\boldsymbol{r}, \boldsymbol{p}: p \gg p l o w b, t) ; p \lesssim p_{\max }$

with growing $p_{\max }$.

Consider a very simple argument in which the the number of particles in $\left(\boldsymbol{r}_{1}, \boldsymbol{p}_{1}\right)$ is given by the value of the distribution function element of the form $f\left(\boldsymbol{r}_{1}, \boldsymbol{p}_{1}: p_{1} \lesssim\right.$ $\left.p_{\max }, t\right)=10^{n}$. After a time $\tau=\Delta t$ this number of particles is reduced:

$$
f\left(\boldsymbol{r}_{1}^{\prime}, \boldsymbol{p}_{1}^{\prime}: p_{1}^{\prime} \lesssim p_{\max }^{\prime}, t+\tau\right)=10^{n} \exp \left[-\lambda^{\prime}\left(\boldsymbol{r}, \boldsymbol{p}_{\max }, t\right) \tau\right] .
$$

The e-folding time for this decay can be expressed in the form $\tau=2.3 n / \lambda^{\prime}\left(\boldsymbol{r}, \boldsymbol{p}_{\max }, t\right)$, which we can identify as the characteristic time for the collapse of the high energy tail of the distribution function. This gives ample time for $p \lesssim \boldsymbol{p}_{\max }$ to grow along an orbit before the distribution function collapses because when $p_{\max }<p_{\mathrm{b}}, \lambda^{\prime}\left(\boldsymbol{r}, \boldsymbol{p}_{\max }, t\right)=0$, and $\tau=\infty$; but when $p_{\max }>p_{\mathrm{b}}, \lambda^{\prime}\left(\boldsymbol{r}, \boldsymbol{p}_{\max }, t\right)>0$ the value of $\tau \rightarrow 2.3 n / \lambda\left(\boldsymbol{r}, \boldsymbol{p}_{\max }, t\right)$, a very small value, because $\lambda^{\prime}\left(\boldsymbol{r}, \boldsymbol{p}_{\max }, t\right) \rightarrow \lambda\left(\boldsymbol{r}, \boldsymbol{p}_{\text {max }}, t\right)$, as a consequence of the widening of the interval $\left(\omega_{\mathrm{b}}, \omega_{\max }\right)$ with growing $p_{\max }$.

Let us consider the evolution of a set of particles at position $\boldsymbol{r}$, with momentum $p$ at time $t$. Then the change of energy of this set of particles is

$$
d \mathcal{E}=e(\boldsymbol{E} \cdot \boldsymbol{v}) d t-\hbar \omega
$$

where $\omega \in\left(0, \omega_{\max }\right)$ and $d t$ are random variables. Than averaging in time and frequency, we get

$$
\langle d \mathcal{E}\rangle=P_{\text {Lor }}\langle d t\rangle-\hbar\langle\omega\rangle
$$

As $P_{\text {Rad }}=\hbar \lambda(\boldsymbol{r}, \boldsymbol{p}, t)\langle\omega\rangle$ and assuming $\langle d t\rangle \approx$ $[\lambda(\boldsymbol{r}, \boldsymbol{p}, t)]^{-1}$, we get

$$
\frac{\langle d \mathcal{E}\rangle}{\langle d t\rangle}=P_{\text {Lor }}-P_{\mathrm{Rad}}
$$

which we immediately recognise as similar to the 4-term of the L-DEM, with $P_{\mathrm{Rad}}=\hbar \lambda(\boldsymbol{r}, \boldsymbol{p}, t)\langle\omega\rangle$. But now such expression has a complete different meaning. When we have a set of particles in phase space at position $(\boldsymbol{r}, \boldsymbol{p})$ they move to a set of cells

$$
\begin{aligned}
\left\{\boldsymbol{r}+d \boldsymbol{r}, \boldsymbol{p}^{\prime}: p^{\prime} \in\right. & {\left[p+\left(P_{\text {Lor }} d t-\hbar \omega_{\max }\right) / c,\right.} \\
& p+\left(P_{\text {Lor }} d t-\hbar\langle\omega\rangle\right) / c \\
& \left.\left.p+P_{\text {Lor }} d t / c\right]\right\}
\end{aligned}
$$

provided $\langle\omega\rangle>\omega_{b}$ and therefore there are particles above and below the radiative average. Even if $P_{\text {Lor }} / P_{\text {Rad }} \sim 1$, with $\lambda^{\prime}(\boldsymbol{r}, \boldsymbol{p}, t) \sim \lambda(\boldsymbol{r}, \boldsymbol{p}, t)$ the value of $p_{\max }$ keeps growing, although due to radiative decay slower then when $\left.\lambda^{\prime}\left(\boldsymbol{r}, \boldsymbol{p}_{\max }, t\right) \ll \lambda\left(\boldsymbol{r}, \boldsymbol{p}_{\max }\right), t\right)$, as the new

$$
p_{\text {max }}^{\prime} \in\left(p+\left(P_{\text {Lor }} d t-\hbar\langle\omega\rangle\right) / c, p+P_{\text {Lor }} d t / c\right) .
$$

This is in good agreement the calculations performed in Paper II when compared with da Costa [29]. They show that the dynamical evolution of the plasma ensures that $P_{\text {Lor }} / P_{\text {Rad }} \lesssim 1$, with $\Upsilon \ll 1$, meaning that the radiative time scale never matches the Lorentz time scale for the q-classical regime. When this ratio is reached, $p_{\max }$ can still continue to grow very slowly implying a growing $\Upsilon$, when the plasma flow turns slowly from a q-classical $(\Upsilon \ll 1)$ to a quasi-quantum regime $(\Upsilon \gtrsim 1)$ and we may foresee a recycling process.

In fact when the slow growth of $p_{\max }$ is initiated, $\Upsilon$ grows with $p_{\max }$, and overtakes $\Upsilon \approx 1$. Then the plasma particles start radiating photons almost as energetic as the particles themselves, their very high energy radiative spectrum widens, and for $\Upsilon\left(p \lesssim p_{\max }\right) \gg 1$ their numbers are as large as a growing $\lambda\left(\boldsymbol{r}, \boldsymbol{p}_{\max }, t\right)$, with $p_{\max }$. Then the number of radiated photons per unit time with $\hbar \omega<0.01 m c^{2}$ is very small compared with the overall number of radiated photons. This combined with the higher $\lambda\left(\boldsymbol{r}, \boldsymbol{p}_{\max }, t\right)$ allows a stronger radiative decay, as there are fewer and fewer particles moving from very high values of $p$ moving into closer values of momentum, the consequent collapse of the high-energy plasma, and the appearance of a plasma stream with comparatively little kinetic energy. But at this situation $p_{\max }$ remains stable because although the radiative decay acts to diminish the distribution function tail, the transition from the former q-classical regime and the new q-quantum regime keeps going, and the external fields act to boost the smaller values of momenta $p<p_{\max }$. 
Intrinsically, the production of photons is a discrete process, and we must account for this underlying phenomenon in our description of the evolution of the particle distribution functions. The noise on the photon count $N_{\nu}$ at each frequency $\nu$ is proportional to $N_{\nu}^{-1 / 2}$. Therefore the counts of the highest energy photons have the greatest uncertainty by virtue of their relatively small number. Since the only source of these photons (by model assumption) is the plasma particles, then radiation processes must produce greater noise on the high-energy tail of the distribution function than elsewhere. This effect is most evident when $P_{\text {Lor }} / P_{\text {Rad }} \gtrsim 1$ for $p \sim p_{\max }$, and therefore we might expect the formation of very strong random perturbations in the distribution function $f(\boldsymbol{r}, \boldsymbol{p}, t)$.

Such disturbances will give rise to charged particle number perturbations (at least) in the rest frame; this activity will in turn generate electrostatic oscillations, and produce electromagnetic waves at frequencies that are multiples of the local plasma frequency as a direct result of mode coupling (da Costa, Diver \& Stewart [21]; Stark et al. [35]). Adding these fields to the underlying electromagnetic field, the value of $\Upsilon$ in the laboratory frame can grow very steeply and suddenly, to $\Upsilon \gg 1$, producing the effects described in the previous paragraph. In this way the collective plasma behaviour feeds back into the radiation process.

The radiation of high energy photons with energies much greater than the ones for $P_{\text {Lor }} / P_{\text {Rad }} \sim 1$ is possible, but given that the ultra-high energy particles that are the source of such photons must be the tiny minority confined to the high-energy tail of the distribution function around $p_{\max }$, then these photons are necessarily much fewer in number than the bulk of the radiation. There is however the possibility that these ultra highenergy photons produce pairs (either by interacting with the ambient magnetic field, or by photon-photon interaction) and therefore these ultra-high energy photons will survive only in very small numbers compared with their less energetic counterparts.

Finally, note that when $p_{\max } \geq p_{b}$, then the distribution function has a double character: the high-energy component is either q-classical or q-quantum, radiating strongly, but as a result, there is a spread of lower momenta which should be considered to be classical in nature. Populating the lower momentum states as a direct result of radiation processes will in general lead to a double-peaked distribution function; such a form cannot be considered to be an equilibrium, and indeed the presence of a 'saddle point' in momentum space must lead to plasma instabilities (such as the onset of plasma oscillations).

This means that a relativistic plasma, even if starts being in dynamic equilibrium with the environment, after reaching a certain value of $\gamma$ and starting to radiate strongly, becomes quite unstable, never coming again to terms with the environment in which it is immersed.

\section{The evolution of magnetospheric plasmas}

The picture so far developed may be applied to pulsar magnetospheres, excluding the region in the immediate vicinity of the star (the atmosphere).

In pulsar magnetospheres the source of plasma is the surface of the star, which is rotating. It is usual to use a mathematical rotating frame of reference, with azimuthal coordinate $\psi \equiv \phi-\omega t$, to give the quasi-static condition in which $\psi$ can be used instead of $t$. As before we assume that the plasma is collisionless, since collisional effects are largely irrelevant for the dynamics of the magnetosphere.

For the low-energy plasma, with no radiative effects, the Vlasov description is acceptable, when adapted for the mathematical rotating frame of reference. Particle trajectories are more correctly described as pseudotrajectories in the rotating frame of reference, and they form a 6-manifold hypersurface in phase space, as before. These pseudo-trajectories in the rotating frame are patterns which rotate with the star in the laboratory frame, and are very close to the magnetic field lines. In the absence of an electric field component parallel to the magnetic field, this would be a pure magnetic slingshot; however, this picture is perturbed by the presence of an $E_{\|}=(\boldsymbol{E} \cdot \boldsymbol{B}) / B$ component.

As seen in Section I the pulsar EM field is a combination of two fields: (1) the star's quasi-static fixed dipolar EM field, described by the Deutsch Model and (2) the time-dependent, non-quasi-static fields generated by the creation of charge and current density distributions by the flow itself and other EM plasma self-fields generated by radiative processes (when outside the SLC, this is known as the Schott field as described in [23, 36]), influenced by the effort to minimize the value of the global $\boldsymbol{E} \cdot \boldsymbol{B} / B \neq 0$ (Le Chatelier Principle [37].

With these provisos that are particular to the pulsar environment, we may now carry over all the physics from the previous sections and apply it to the pulsar magnetosphere. We assume the electron-positron magnetospheric plasma leaves the quantum region around the star with very low energy (typically $\gamma<10$ ) because, inside this region the EM fields are so strong that particles lose energy from radiation of high energy gamma-ray photons (as energetic as the particles which radiated them). These high energy photons produce pairs, augmenting the plasma density. This low-energy plasma will find itself far from radiative conditions, and is accelerated by the low frequency EM field, following closely the magnetic field lines. Thus the accelerated pair plasma at some critical distance out from the pulsar may depart significantly from motion parallel to the local magnetic field in the rotating frame (in the vicinity of the SLC, but the geometry is complex), and by virtue of the energy it attained, enters the q-classical regime.

In this regime, when the energy acquired between the radiation of two successive photons is of the same order of magnitude as the energy of the radiated photons, a nearbalance is established, with $\gamma$ continues growing now very 
slowly. This is now the regime in which plasma recycling is dominant, and the upper value of radiated photons is well above the $10-50 \mathrm{GeV}$ range, where $P_{\text {Lor }} / P_{\text {Rad }} \sim 1$. Such strong radiation quenching of momenta provokes the collapse of the high-energy plasma. This means that the particles are accelerated until a certain value of energy, and through radiation the particle loses almost all its energy, and that $f(\boldsymbol{r}, \boldsymbol{p}, t)=0$ for $p>p_{\max }$. The particles return to the classical domain, and because it is co-spatial with higher energy plasma, an instability must develop.

This reasoning is supported by the particle pseudotrajectory approach and the calculation performed before in Paper II, which show that it is very unlikely that the energy of the particles will reach very high energies. The Lorentz force in the plasma will have an upper limit $\gamma \sim$ $10^{9}$ and therefore photons with energies above $50 \mathrm{GeV}$ will be scarce, and even depleted by pair production, and no energetic photons with $\hbar \omega>50 \mathrm{TeV}$ will be emitted.

\section{The vacuum polarization problem and Curve-Cerenkov radiation}

The radiation processes described above are not the only such processes that may be present in the plasma. The vacuum in the presence of a magnetic field polarizes and therefore its refractive index is [31], assuming the angle between the trajectory an the magnetic field lines $\theta=\pi / 2$

$$
\begin{aligned}
& n_{\|}=1+\kappa_{1} \delta \\
& n_{\perp}=1+\kappa_{2} \delta
\end{aligned}
$$

with

$$
\begin{aligned}
\delta & =0.5 \times 10^{-4}\left(B / B_{C l}\right)^{2} \\
B_{C l} & =4.46 \times 10^{9} \mathrm{~T} \\
0<\kappa_{1,2} & <1
\end{aligned}
$$

Assuming for simplicity that $\kappa_{1,2}=1$, then $v=c / n \sim$ $c(1-\delta)$ where $n$ is the isotropic refractive index. Hence if $\gamma \gg 1$, then it is possible that we have curve-Cerenkov Radiation in vacuum $(v>c / n)$ provided $\gamma>\gamma_{c}$, where

$$
\gamma_{c}=(2 \delta)^{-1 / 2}=10^{2}\left(B_{C l} / B\right)
$$

For conditions close to the surface of the star, $B \sim 10^{8} \mathrm{~T}$, and therefore $\gamma_{c} \sim 4 \times 10^{3}$. But close to the SLC, $B \sim 10^{2}$ $\mathrm{T}$, and therefore $\gamma_{c} \sim 4 \times 10^{9}$, if we do not consider the influence of the generated EM fields, due to plasma dynamics. Therefore it is possible that if the value of $\gamma$ goes above the threshold value $\gamma_{c}$ then there are additional radiation losses, and it is necessary to correct the values of radiation parameters in the treatment of the earlier sections. However even before $\gamma$ reaches the critical value, the transition to the onset of curve-Cerenkov radiation changes the local radiation properties.
Such corrections can only be done provided we construct a proper theory of curvature and curve-Cerenkov radiation in anisotropic media - a theory which currently does not exist.

\section{CONCLUSIONS AND FURTHER WORK}

The aim of this paper is to understand the presence of a cut-off in the radiation of high energetic $\gamma$-ray photons, through a self-consistent treatment of high-energy radiation processes in the pulsar environment. In so doing, we have shown where the classical model is deficient in two respects: (1) the lack of self-consistency even at the $25 \mathrm{GeV}$ energy; and (2) the inability to produce $\mathrm{TeV}$ photons in the pulsar context. These deficiencies are corrected in this treatment, abandoning the classical idea that the cut-off has to be at a frequency such that $P_{\text {Rad }} \sim P_{\text {Lor }}$, just in the classical context, as in $[6,7]$, but at a point where $\hbar \omega_{\mathrm{c}} \rightarrow \gamma m c^{2}$ which forces us to consider a q-quantum magnetospheric description. This, we believe, will be a valuable progressive refinement for observers using the latest technology, where the level of technical precision is significantly in advance of the predictive power of the legacy modelling.

The radiation properties described in this paper lead us to conclude that there are two distinct (spatial) regions in the pulsar environment in which ultra-strong radiation processes occur: (1) close to the pulsar surface, where the EM field strengths are so high that particles ejected from the surface radiate photons which very quickly produce electron-positron pairs; and (2) close to the SLC where the secondary plasma created by photon absorption in region (1) has acquired sufficient energy to undergo ultra-strong radiation once more. It might be helpful to consider the first strong radiation location as the boundary of the pulsar atmosphere, and the second to mark the outer edge of the pulsar magnetosphere.

In fact close to the SLC the high-energy plasma reaches a balance with a dynamical radiation process, and the photon energy is kept in the range $10-50 \mathrm{GeV}$. This region is much larger than the region where higher energy photons are radiated, and the depletion of photons through pair-production ensures a very low level above that range, which as discussed, it can never go beyond $50 \mathrm{TeV}$.

To construct a full pulsar $\gamma$-ray spectrum it is required to full model the pulsar along the lines developed in this paper, and therefore to understand the plasma flow in the magnetosphere. The associated kinetic equation for radiative plasmas developed in this paper (absolutely necessary for such study) is a generalization of Vlasov theory, in which the radiative term is added as a 'collisional' contribution to the otherwise zero right-hand side of the kinetic equation. In fact, for relatively low radiation energies, this radiation term is set precisely to zero.

The treatment in this paper is not comprehensive: there are additional physical aspects that may well merit further attention, for example, quantum considerations 
such as: (a) the random effects of radiation due to spin of particles; and (b) the perturbations of motion due to radiation of photons in the direction perpendicular to the plane of trajectory; these effects might turn classical trajectories into quantum ones, forcing the redesign of the kinetic theory. In addition, a fuller theory of curvature and curve-Cerenkov radiation in anisotropic media is clearly needed, in order to arrive at a more complete and satisfactory radiation description. A more complete treatment of the instabilities in the distribution function would provide greater detail about the possible relaxation processes that must be present on short timescales in the non-linear kinetic theory (such as the production of Bernstein modes and streaming instabilities) requiring a kinetic treatment along the lines of da Costa, Diver \& Stewart [21].

\section{ACKNOWLEDGEMENTS}

DAD gratefully acknowledges funding from the UK Science and Technology Funding Council
(STFC/F002149/I), as does CRS (PP/E001122/1). AAdaC is grateful to the Fundação para a Ciência e Tecnologia, Portugal, who sponsored his sabbatical leave, under Grant SFRH/BSAB/771/2007; thanks are due equally to the Instituto Superior Técnico and to the Department of Physics and Astronomy, University of Glasgow, for respectively granting and hosting AAdaC's sabbatical leave. LFAT is grateful to the Leverhulme trust for funding at Glasgow University. Finally, thanks are due to $\mathrm{H}$ E Potts, C S Maclachlan and E Bennet for their helpful comments and stimulating discussion, and to an anonymous referee whose helpful comments helped to shape this paper. The Department of Physics and Astronomy in the University of Glasgow is a member of the Scottish Universities Physics Alliance (SUPA).
[1] E. Aliu \& al, Science 322, 1221 (2008).

[2] R. P. Johnson and R. Mukherjee, New Journal of Physics, 11(5), 055008 (2009).

[3] R. Campana, E. Massaro, T. Mineo \& G. Cusumano, Astron. Astrophys. 499, 847-850 (2009).

[4] J. Takata \& H.-K. Chang, Astrophys. J. 670, 677-692 (2007).

[5] A. Harding and D. Lai, Reports on Progress in Physics 69, 2631-2708 (2006).

[6] M. G. Higgins and R. N. Henriksen, Mon. Not. R. Astron. Soc. 292, 934-944 (1997).

[7] M. G. Higgins and R. N. Henriksen, Mon. Not. R. Astron. Soc. 295, 188-196 (1998).

[8] C. Kalapotharakos and I. Contopoulos, Astron \& Astrophysics 496, 495-502 (2009).

[9] A. P. S. Tang, J. Takata, J. Jia \& K. S. Cheng, Astrophys. J. 676, 562-572 (2008).

[10] A. Gruzinov, Astrophys. J. Lett. 667, L69-L71, (2007).

[11] T. Wada \& S. Shibata, Mon. Not. R. Astron. Soc. 376 1460-1464 (2007).

[12] P. Goldreich and W. H. Julian, Astrophys. J. 157, 869880 (1969).

[13] L. Mestel, Nuture 233, 149 (1971).

[14] L. Mestel, P. Panagi \& S. Shibata, Mon. Not. R. Astron. Soc. 309, 388-394 (1999).

[15] J. M. Cohen and E. T. Toton, Astrophys. J. Lett. 7, 213-215 (1971).

[16] A. J. Deutsch, Annales d'Astrophysique 18, 1-10 (1955).

[17] T. J. M. Boyd and J. J. Sanderson, The Physics of Plasmas, Cambridge University Press, Cambridge, 2003.

[18] A. A. da Costa and F. D. Kahn, Mon. Not. R. Astron. Soc. 199, 211-217 (1982).

[19] R. Fitzpatrick and L. Mestel, Mon. Not. R. Astron. Soc. 232, 277-302 (1988).

[20] R. Fitzpatrick and L. Mestel, Mon. Not. R. Astron. Soc.
232, 303-321 (1988).

[21] A. A. da Costa, D. A. Diver and G. A. Stewart, Astron. Astrophys. 366, 129-137 (1994).

[22] E. T. Rowe, Astron. Astrophys. 296, 275-288 (1995).

[23] A. A. da Costa and F. D. Kahn, Mon. Not. R. Astron. Soc. 199, 701-711 (1985).

[24] F. D. Kahn, Paper read at a RAS Meeting, unpublished, February, 1971.

[25] P. A. M. Dirac, Proceedings of the Royal Society of London A 167, 148-168 (1938).

[26] L. D . Landau and E. M. Lifshitz, The Classical Theory of the Fields. Landau and Lifshitz Course of Theoretical Physics, volume 2, Elsevier, Amsterdam, 2008.

[27] J. Schwinger, Phy. Rev. 75 (12), 1912-1925 (1949).

[28] J. D . Jackson, Classical Electrodynamics. John Wiley \& Sons, Inc., New York, 1999.

[29] A. A. da Costa, Mon. Not. R. Astron. Soc. 204, 11251144 (1983).

[30] A. A. da Costa and F. D. Kahn, Mon. Not. R. Astron. Soc. 284, 681-686 (1991).

[31] P. Mészáros, High-Energy Radiation from Magnetized Neutron Stars. The University of Chicago Press, Chicago, 1992.

[32] D. L. Snider and M. I. Miller, Springer, Berlin, 1991.

[33] A. A. Sokolov and I. M. Ternov. Radiation from Relativistic Electrons. AIP Translation Series. American Institute of Physics, New York, 1986.

[34] V. B . Berestetskii, E. M. Lifshitz and L. P. Pitaevskii. Quantum Electrodynamics. Landau and Lifshitz Course of Theoretical Physics, volume 4, Pergamon Press, Oxford, 1980.

[35] C. R. Stark, D. A. Diver, A. A. da Costa and E. W. Laing, Astron. Astrophys. 476, 17-30 (2007).

[36] A. A. da Costa and F. D. Kahn, Mon. Not. R. Astron. Soc. 284, 1-26 (1997). 
Symbol first defining equation description

\begin{tabular}{lll}
\hline \hline$\xi$ & $(1)$ & radiation coefficient \\
$P_{\text {Lor }}$ & $(2)$ & Power generated by Lorentz force \\
$P_{\text {Dir }}$ & $(2)$ & Power in classical radiation term \\
$P_{r}$ & $(15)$ & Radiation power spectrum \\
$P_{p}$ & $=P_{r} /(\hbar \omega)$ & normalised radiation power spectrum \\
$P_{\text {rad }}$ & $(28)$ & total power radiated \\
$\lambda$ & $(21)$ & average number of radiated photons per unit time \\
$\gamma$ & $=\left(1-v^{2} / c^{2}\right)^{-1 / 2}$ & relativistic factor \\
$\omega_{c}$ & $(7)$ & critical frequency beyond which there is negligible radiation \\
$\omega_{\text {peak }}$ & $(3)$ & frequency at which the peak of the classical synchrotron radiation spectrum occurs \\
$\Omega$ & $(9)$ & angular velocity associated with rotation of charged particles in pulsar fields \\
$\zeta$ & $(38)$ & curvature radiation parameter \\
$F$ & $(13)$ & normalised magnitude of perpendicular component of Lorentz force \\
$p_{q c l}$ & $\omega\left(p_{q c l}\right)=m c^{2} / \hbar$ & transition point in momentum space where radiated photon energy is of order of total energy \\
$\Upsilon$ & $(34)$ & curvature radiation parameter that discriminates between classical and non-classical regimes \\
\hline \hline
\end{tabular}

[37] L. Mestel,G. A. E. Wright, and K. C. Westfold, Mon. Not. R. Astron. Soc. 175, 257-278 (1976).

[38] F. R. D. Agudo, Lições de Análise Infinitesimal: I. Cálculo Diferencial em $R^{n}$. Livraria Escolar Editora, Lisboa 1969.

[39] H. Ardavan, Nature 289, 44-45 (1981).

[40] A. A. da Costa, Astrophysics and Space Science 216, 179-184 (1994).

[41] Zhang Jia-Lú and K. S. Cheng, Chin. Astron. Astrophys. 20(2), 239-243 (1996).

[42] C. W. Gardiner, Handbook of stochastic Methods, for Physics, Chemistry and the Natural Sciences. SpringerVerlag, Berlin, 1983.

[43] D. R. Nicholson, Introduction to Plasma Theory. John
Wiley \& Sons, New York, 1983.

[44] F. D . Kahn and A. A. da Costa, Proceedings of the NATO Advanced Research Workshop on Cosmical Magnetism in honour of Leon Mestel, Cambridge, UK, July 5-9 1993, D. Lynden Bell (ed.), 99-111. Kluwer Academic Publishers, Dordrecht, 1994.

\section{Appendix A: Notation}

A guide to the notation used in this article is included in Table I for the convenience of the reader.

\section{Appendix B: Curvature Radiation versus Synchrotron Radiation}

There is a similarity between synchrotron and curvature radiation, as they have the same power law, when expressed in terms of the radius of curvature of their associated trajectories [28, 41], periodic (circular) in the case od synchrotron radiation and non-periodic in the case of curvature radiation. In this way synchrotron radiation is periodic curvature radiation.

From the theory of synchrotron radiation we know that the observed pulse comes from a limited section of the trajectory, repeating itself with period $T$, and the same must be true for curvature radiation [28], without repetition. Let us suppose that the non-periodic trajectory is given by $\boldsymbol{r}(t)$, and than

$$
\boldsymbol{r}(t)=\int \boldsymbol{r}(\tau) \delta(t-\tau) d \tau,
$$

with a corresponding power spectrum $P(\omega)$, which is a function of the radius of curvature. But if the trajectory is periodic than the trajectory is given by

$$
\boldsymbol{r}_{p}(t)=\int \boldsymbol{r}(\tau) \Delta(t-\tau) d \tau
$$

where

$$
\Delta(t)=\sum_{n} \delta(t-n T)
$$

whose Fourier transform

$$
\mathcal{F}[\Delta(t)]=\Delta(\omega)=\sum_{n} \delta\left(\omega-n \omega_{0}\right),
$$


being $\omega_{0}=2 \pi / T$, and the corresponding power spectrum generated by the periodic trajectory $P(\omega) \Delta(\omega)$. This is a discrete spectrum but with an underlying power law similar to the non-periodic one.

The similarity in power law, expressed in terms of radius of curvature, between synchrotron and curvature radiation is broken when we take into account the different radii of curvature: in the case of synchrotron radiation is given by expression (10), and for curvature radiation by (9). Expression (10) is indeed a special case of (9), when $\boldsymbol{E}=\mathbf{0}$.

Expression (9) may be found as follows. The radius of curvature of the trajectory $\boldsymbol{r}(t)$ is [38]

$$
\frac{1}{\rho}=\frac{|\dot{\boldsymbol{r}} \times \ddot{\boldsymbol{r}}|}{\dot{r}^{3}}
$$

and as

$$
\dot{\boldsymbol{r}}=\frac{c \boldsymbol{p}}{\sqrt{m^{2} c^{2}+p^{2}}}
$$

than taking into account that for $\gamma \gg 1, p \sim m c \gamma \gg m c$,

$$
\frac{1}{\rho}=\frac{|\boldsymbol{p} \times \dot{\boldsymbol{p}}|}{c p^{2}}
$$

and using Lorentz equation $\dot{\boldsymbol{p}}=e(\boldsymbol{E}+\boldsymbol{v} \times \boldsymbol{B})$,

$$
\frac{1}{\rho}=e \frac{|\boldsymbol{p} \times(\boldsymbol{E}+\boldsymbol{v} \times \boldsymbol{B})|}{c p^{2}}
$$

and (9) is immediately found. When $\boldsymbol{E}=\mathbf{0}$, taking into account that $v \sim c$, equation (10) is also immediately found.

\section{Appendix C: The relativistic kinetic Theory of relativistic plasmas}

We intend to establish the kinetic equation to describe the evolution of a collisionless plasma. Since this radiative phenomenon is a compound Markov process, a Cauchy process, we shall follow the Gardiner treatment [42].These collisionless plasmas are immersed in an EM field such that radiative effects have to be considered only when $\gamma \gg 1$, which means that there is a boundary classical $\Longleftrightarrow$ q-classical regimes defined by relations $(25)$ and $\Upsilon \ll 1$. We are aiming for an extension of the Vlasov equation, no microstability collisional analysis is considered, and therefore the Fokker-Planck terms will be omitted.

\section{Basic Principles}

Cauchy processes are discontinuous Markov processes and therefore they obey the Chapman-Kolmogorov equation

$$
p\left(\boldsymbol{x}_{1}, t_{1} \mid \boldsymbol{x}_{3}, t_{3}\right)=\int d \boldsymbol{x}_{2} p\left(\boldsymbol{x}_{1}, t_{1} \mid \boldsymbol{x}_{2}, t_{2}\right) p\left(\boldsymbol{x}_{2}, t_{2} \mid \boldsymbol{x}_{3}, t_{3}\right)
$$

which means that the probability of a process reaching $\left(\boldsymbol{x}_{1}, t_{1}\right)$ after departing from $\left(\boldsymbol{x}_{3}, t_{3}\right)$ depends on all intermediate positions $\left(\boldsymbol{x}_{2}, t_{2}\right)$. Nevertheless Cauchy processes do not obey the Lindeberg equation

$$
\lim _{\Delta t \rightarrow 0} \frac{1}{\Delta t} \int_{|\boldsymbol{x}-\boldsymbol{z}|>\epsilon} d \boldsymbol{x} p(\boldsymbol{x}, t+\Delta t \mid \boldsymbol{z}, t)=0
$$

due to the discrete nature of the trajectory considered in a Cauchy process, as the probability that the final position $\boldsymbol{x}$ to be finitely different from $\boldsymbol{z}$ either goes to zero much slower than $\Delta t$ or may be even finite [42]. In the Lindeberg Equation $\epsilon$ never vanishes, although it goes to zero, such that we never lose the basis for the Markov condition. $\Delta t$ and $\epsilon$ are interrelated, and when one is infinitesimal, the other follows.

As the Lindeberg equation is not satisfied, we may put

$$
p(\boldsymbol{x}, t+\Delta t \mid \boldsymbol{z}, t)=p_{\Delta t}(\boldsymbol{x} \mid \boldsymbol{z})
$$


and in this case following the Chapman-Kolmogorov equation

$$
p\left(\boldsymbol{x}_{1}, t_{1} \mid \boldsymbol{x}_{3}, t_{1}-\Delta t\right)=\int d \boldsymbol{z} p\left(\boldsymbol{x}_{1}, t_{1} \mid \boldsymbol{z}, t_{1}-\Delta t\right) p\left(\boldsymbol{z}, t_{1}-\Delta t \mid \boldsymbol{x}_{3}, t_{1}-\Delta t\right)
$$

then

$$
\begin{aligned}
p\left(\boldsymbol{x}_{1}, t_{1} \mid \boldsymbol{x}_{3}, t_{1}\right) & =\lim _{\Delta t \rightarrow 0} \int d \boldsymbol{z} p_{\Delta t}(\boldsymbol{x} \mid \boldsymbol{z}) p\left(\boldsymbol{z}, t_{1}-\Delta t \mid \boldsymbol{x}_{3}, t_{1}-\Delta t\right) \\
& =\int d \boldsymbol{z} p_{\Delta t}(\boldsymbol{x} \mid \boldsymbol{z}) p\left(\boldsymbol{z}, t_{1} \mid \boldsymbol{x}_{3}, t_{1}\right)
\end{aligned}
$$

and therefore we might identify

$$
\lim _{\Delta t \rightarrow 0} p(\boldsymbol{x}, t+\Delta t \mid \boldsymbol{z}, t)=\delta(\boldsymbol{x}-\boldsymbol{z})
$$

where $\delta(\boldsymbol{x}-\boldsymbol{z})$ is the Dirac delta function. Following similar considerations:

1. $\lim _{\Delta t \rightarrow 0} p(\boldsymbol{x}, t+\delta t \mid \boldsymbol{z}, t) / \Delta t=W(\boldsymbol{x} \mid \boldsymbol{z}, t)$ for $|\boldsymbol{x}-\boldsymbol{z}|>z$

2. $\lim _{\Delta t \rightarrow 0} \frac{1}{\Delta t} \int_{|\boldsymbol{x}-\boldsymbol{z}|<z} d \boldsymbol{x}\left(x_{i}-z_{i}\right) p(\boldsymbol{x}, t+\delta t \mid \boldsymbol{z}, t)=A_{i}(\boldsymbol{z}, t)+O(\epsilon)$

3. $\lim _{\Delta t \rightarrow 0} \frac{1}{\Delta t} \int_{|\boldsymbol{x}-\boldsymbol{z}|<z} d \boldsymbol{x}\left(x_{i}-z_{i}\right)\left(x_{j}-z_{j}\right) p(\boldsymbol{x}, t+\delta t \mid \boldsymbol{z}, t)=B_{i j}(\boldsymbol{z}, t)+O(\epsilon)$

All other coefficients $C_{i j \ldots l}=O(\epsilon)$ and therefore $\lim _{\Delta t \rightarrow 0} C_{i j \ldots l}=0$

Using these considerations we try to establish a differential equation, as a counterpart of the Chapman-Kolmogorov equation, taking into account all kinds of Markov processes present. We shall arrive at the differential Gardiner Equation

$$
\frac{\partial p(\boldsymbol{x}, t)}{\partial t}=-\nabla \cdot[\boldsymbol{A}(\boldsymbol{x}, t) p(\boldsymbol{x}, t)]+\frac{1}{2} \nabla \nabla:[\boldsymbol{B}(\boldsymbol{x}, t) p(\boldsymbol{x}, t)]+K_{\text {rad }}(\boldsymbol{x}, t)
$$

where we have changed $p\left(\boldsymbol{x}, t \mid \boldsymbol{x}_{i n}, t_{i n}\right)$ into $p(\boldsymbol{x}, t)$, assuming $\left(\boldsymbol{x}_{i n}, t_{i n}\right)$ the starting configuration [43], with

$$
K_{\text {rad }}(\boldsymbol{x}, t)=\int d \boldsymbol{x}\left[W\left(\boldsymbol{x} \mid \boldsymbol{x}_{1}, t\right) p\left(\boldsymbol{x}_{1}, t\right)-W\left(\boldsymbol{x}_{1} \mid \boldsymbol{x}, t\right) p(\boldsymbol{x}, t)\right]
$$

\section{The kinetic equation}

The term $K_{\text {rad }}(\boldsymbol{x}, t)$ gives notice of transitions $\boldsymbol{x}_{1} \rightarrow \boldsymbol{x}$ and $\boldsymbol{x} \rightarrow \boldsymbol{x}_{1}$. However in plasmas we may put $\boldsymbol{x}=(\boldsymbol{r}, \boldsymbol{p})$ and the Cauchy process occurs at fixed $\boldsymbol{r}$ from $\boldsymbol{p}_{1} \rightarrow \boldsymbol{p}$ and $\boldsymbol{p} \rightarrow \boldsymbol{p}_{1}$. Therefore putting $\boldsymbol{y}=(\boldsymbol{q}, \boldsymbol{s})$

$$
W(\boldsymbol{x} \mid \boldsymbol{y}, t)=\delta(\boldsymbol{r}-\boldsymbol{q}) W(\boldsymbol{r}, \boldsymbol{p} \mid \boldsymbol{s}, t)
$$

In an associated kinetic description we change $p(\boldsymbol{x}, t)$ into $f(\boldsymbol{r}, \boldsymbol{p}, t), \boldsymbol{A}(\boldsymbol{x}, t)=(\boldsymbol{v}, \dot{\boldsymbol{p}})$. Therefore making these changes the differential Gardiner equation will assume the more standard form

$$
\frac{D f}{D t} \equiv \frac{\partial f}{\partial t}+\boldsymbol{v} \cdot \nabla f+\boldsymbol{F} \cdot \nabla_{p} f=\left(\frac{\delta f}{\delta t}\right)_{\substack{\text { spont } \\ \text { emiss }}}
$$

In this case

$$
\left(\frac{\delta f}{\delta t}\right)_{\substack{\text { spont } \\ \text { emiss }}}=\int d \boldsymbol{p}_{1}\left[W\left(\boldsymbol{r}, \boldsymbol{p} \mid \boldsymbol{p}_{1}, t\right) f\left(\boldsymbol{r}, \boldsymbol{p}_{1}, t\right)-W\left(\boldsymbol{r}, \boldsymbol{p}_{1} \mid \boldsymbol{p}, t\right) f(\boldsymbol{r}, \boldsymbol{p}, t)\right]
$$

In the above integral everything happens in fixed position in configuration space $(\boldsymbol{r}, t)$. The first term gives the density of particles per unit time which access $\boldsymbol{p}$. At the same time the second term gives the density of particles per unit time which are available to leave $\boldsymbol{p}$. 
$W\left(\boldsymbol{r}, \boldsymbol{p}_{\alpha} \mid \boldsymbol{p}_{\beta}, t\right)$ is the probability per unit time that a particle will jump from $\boldsymbol{p}_{\beta} \rightarrow \boldsymbol{p}_{\alpha}$ at a given position $\boldsymbol{r}$ and time $t[42] . W\left(\boldsymbol{r}, \boldsymbol{p}_{\alpha} \mid \boldsymbol{p}_{\beta}, t\right)=0$ for $\boldsymbol{p}_{\beta} \rightarrow \boldsymbol{p}_{\alpha}$ not allowed transitions. Then

$$
W\left(\boldsymbol{r}, \boldsymbol{p}_{\alpha} \mid \boldsymbol{p}_{\beta}, t\right)= \begin{cases}P_{p n}\left(\boldsymbol{r}, \boldsymbol{p}_{\beta}, \omega, \boldsymbol{n}, t\right)\left\{\frac{\partial[\boldsymbol{p}=(\hbar \omega / c) \boldsymbol{n}]}{\partial(\omega, \boldsymbol{n})}\right\}^{-1}, & \text { for allowed } \boldsymbol{p}_{\beta} \rightarrow \boldsymbol{p}_{\alpha}, p_{\beta}>p_{\alpha} \\ 0, & \text { otherwise. }\end{cases}
$$

with $\boldsymbol{p}_{\beta}=\boldsymbol{p}_{\alpha}+(\hbar \omega / c) \boldsymbol{n}$.

So far all these expressions apply provided there is a $\boldsymbol{p}_{\max }, p_{\max }>p_{\mathrm{qcl}}$ such that

$$
f(\boldsymbol{r}, \boldsymbol{p}, t)=\left\{\begin{array}{l}
\neq 0, \quad p<p_{\max } \\
\sim 0, \text { otherwise }
\end{array}\right.
$$

because in the classical domain

$$
f(\boldsymbol{r}, \boldsymbol{p}, t)=\left\{\begin{array}{l}
\neq 0, p_{\min }<p<p_{\max } \\
\sim 0, \text { otherwise }
\end{array}\right.
$$

The density of particles which will be available for radiation at $\boldsymbol{p}$ per unit time is

$$
\begin{aligned}
\mathcal{N}(\boldsymbol{r}, \boldsymbol{p}, t) & =\int d \boldsymbol{p}_{1} W\left(\boldsymbol{r}, \boldsymbol{p}_{1} \mid \boldsymbol{p}, t\right) f(\boldsymbol{r}, \boldsymbol{p}, t) \\
& =\lambda(\boldsymbol{r}, \boldsymbol{p}, t) f(\boldsymbol{r}, \boldsymbol{p}, t)
\end{aligned}
$$

Then $(\delta f / \delta t)_{\substack{\text { spont } \\ \text { emiss }}}^{\text {f }} \neq 0$, when $\boldsymbol{p}_{\max }$ is in the q-classical domain, and $f(\boldsymbol{r}, \boldsymbol{p}, t) \neq 0$. Using all the available information and calling

$$
\begin{aligned}
\mathcal{N}_{c}(\boldsymbol{r}, \boldsymbol{p}, t) & =\int_{\left|\boldsymbol{p}_{1}\right|>m c} d \boldsymbol{p}_{1} W\left(\boldsymbol{r}, \boldsymbol{p} \mid \boldsymbol{p}_{1}, t\right) f\left(\boldsymbol{r}, \boldsymbol{p}_{1}, t\right) \\
& =\int_{0}^{\omega_{\max }} d \boldsymbol{n} d \omega P_{p n}\left(\boldsymbol{r}, \boldsymbol{p}+\frac{\hbar \omega}{c} \boldsymbol{n}, \omega, \boldsymbol{n}, t\right) f\left(\boldsymbol{r}, \boldsymbol{p}+\frac{\hbar \omega}{c} \boldsymbol{n}, t\right)
\end{aligned}
$$

where $c \boldsymbol{p}+\hbar \omega_{\max } \boldsymbol{n}=c \boldsymbol{p}_{\max }$, we may write

$$
\left(\frac{\delta f}{\delta t}\right)_{\substack{s p o n t \\ \text { emiss }}}=\int_{0}^{\omega_{\max }} d \boldsymbol{n} d \omega P_{p m}\left(\boldsymbol{r}, \boldsymbol{p}+\frac{\hbar \omega}{c} \boldsymbol{n}, \omega, \boldsymbol{n}, t\right) f\left(\boldsymbol{r}, \boldsymbol{p}+\frac{\hbar \omega}{c} \boldsymbol{n}, t\right)-\lambda(\boldsymbol{r}, \boldsymbol{p}, t) f(\boldsymbol{r}, \boldsymbol{p}, t)
$$

$\boldsymbol{n}$ is centered around $\boldsymbol{p}+(\hbar \omega / c) \boldsymbol{n}$, and the aperture angle of the radiating cone is such that

$$
\theta \approx \sin \theta=\frac{|[\boldsymbol{p}+(\hbar \omega / c) \boldsymbol{n}] \times \boldsymbol{n}|}{|\boldsymbol{p}+(\hbar \omega / c) \boldsymbol{n}|} \sim \frac{1}{\gamma} \ll 1
$$

However despite this the angle between $\boldsymbol{p}$ and $\boldsymbol{p}+(\hbar \omega / c) \boldsymbol{n}$ may not be so small, when $\hbar \omega \lesssim \gamma$, and therefore after the radiation the direction of motion night change an angle

$$
\vartheta=\sin ^{-1}\left(\frac{|\boldsymbol{p} \times \boldsymbol{n}|}{p}\right)
$$

This might create several plasma flows at the same point.

\section{The evolutionary process: the solutions}

Let us consider then a plasma leaving a volume through its boundary into a region with a very global strong EM field and in this case this plasma will be far from radiative conditions. Then the equation is the Vlasov equation

$$
\frac{D f}{D t}=0
$$


and the plasma may be considered a cold plasma. However there is a formal equivalence of the Vlasov equation to the equations describing individual particle orbits as seen in Boyd \& Sanderson [17]. These deterministic trajectories form in phase space a 6-manifold hypervolume, which is a network, since

$$
\begin{aligned}
& \boldsymbol{r}_{1}\left(\mathcal{A}_{s}, \boldsymbol{r}, \boldsymbol{p}, \tau\right)=\boldsymbol{r}_{\mathrm{in}_{1}}\left(\mathcal{A}_{s}\right)+\int_{t_{i_{1}}}^{\tau} d \eta \frac{c \boldsymbol{p}_{1}\left[\mathcal{A}_{s}, \boldsymbol{r}_{1}\left(\mathcal{A}_{s}, \boldsymbol{r}, \boldsymbol{p}, \eta\right), \boldsymbol{p}_{1}\left(\mathcal{A}_{s}, \boldsymbol{r}, \boldsymbol{p}, \eta\right), \eta\right]}{\sqrt{m^{2} c^{2}+\boldsymbol{p}_{1}^{2}\left[\mathcal{A}_{s}, \boldsymbol{r}_{1}(\boldsymbol{r}, \boldsymbol{p}, \eta), \boldsymbol{p}_{1}\left(\mathcal{A}_{s}, \boldsymbol{r}, \boldsymbol{p}, \eta\right), \eta\right]}} \\
& \boldsymbol{p}_{1}\left(\mathcal{A}_{s}, \boldsymbol{r}, \boldsymbol{p}, \tau\right)=\boldsymbol{p}_{\mathrm{in}_{1}}\left(\mathcal{A}_{s}\right)+\int_{t_{i_{1}}}^{\tau} d \eta \boldsymbol{F}\left[\boldsymbol{r}_{1}\left(\mathcal{A}_{s}, \boldsymbol{r}, \boldsymbol{p}, \eta\right), \boldsymbol{p}_{1}\left(\mathcal{A}_{s}, \boldsymbol{r}, \boldsymbol{p}, \eta\right), \eta\right]
\end{aligned}
$$

and

$$
\begin{aligned}
& \boldsymbol{r}_{1}\left(\mathcal{A}_{s}, \boldsymbol{r}, \boldsymbol{p}, t\right)=\boldsymbol{r} \\
& \boldsymbol{p}_{1}\left(\mathcal{A}_{s}, \boldsymbol{r}, \boldsymbol{p}, t\right)=\boldsymbol{p}
\end{aligned}
$$

and the distribution function is given by

$$
\left.f_{s}(\boldsymbol{r}, \boldsymbol{p}, t)=\int d \mathcal{A}_{s} g\left(\mathcal{A}_{s}\right) J\left[\boldsymbol{r}_{s}, \boldsymbol{r}_{1}\left(\mathcal{A}_{s}, \boldsymbol{r}, \boldsymbol{p}, t\right)\right)\right] \delta\left[\boldsymbol{r}-\boldsymbol{r}_{1}\left(\mathcal{A}_{s}, \boldsymbol{r}, \boldsymbol{p}, t\right)\right] \delta\left[\boldsymbol{p}-\boldsymbol{p}_{1}\left(\mathcal{A}_{s}, \boldsymbol{r}, \boldsymbol{p}, t\right)\right]
$$

Therefore the integro-differential equation

$$
\frac{D f}{D t}=\mathcal{N}_{c}(\boldsymbol{r}, \boldsymbol{p}, t)-\lambda(\boldsymbol{r}, \boldsymbol{p}, t) f(\boldsymbol{r}, \boldsymbol{p}, t)
$$

may be turned into the Volterra integral equation [44]

$$
\begin{aligned}
f(\boldsymbol{r}, \boldsymbol{p}, t)= & \left.\int d \mathcal{A}_{s} g\left(\mathcal{A}_{s}\right) J\left[\boldsymbol{r}_{s}, \boldsymbol{r}_{1}\left(\mathcal{A}_{s}, \boldsymbol{r}, \boldsymbol{p}, t\right)\right)\right] \delta\left[\boldsymbol{r}-\boldsymbol{r}_{1}\left(\mathcal{A}_{s}, \boldsymbol{r}, \boldsymbol{p}, t\right)\right] \delta\left[\boldsymbol{p}-\boldsymbol{p}_{1}\left(\mathcal{A}_{s}, \boldsymbol{r}, \boldsymbol{p}, t\right)\right] \\
& \exp \left\{-\int_{t_{i n 1}}^{t} d \chi \lambda\left[\boldsymbol{r}_{1}\left(\mathcal{A}_{s}, \boldsymbol{r}, \boldsymbol{p}, \chi\right), \boldsymbol{p}_{1}\left(\mathcal{A}_{s}, \boldsymbol{r}, \boldsymbol{p}, \chi\right), \chi\right]\right\}+ \\
& \int_{t_{\text {in } 2}}^{t} d \eta J\left[\boldsymbol{r}_{2}(\boldsymbol{r}, \boldsymbol{p}, \eta), r\right] \mathcal{N}_{c}\left[\boldsymbol{r}_{2}(\boldsymbol{r}, \boldsymbol{p}, \eta), \boldsymbol{p}_{2}(\boldsymbol{r}, \boldsymbol{p}, \eta), \eta\right] \exp \left\{-\int_{\eta}^{t} d \chi \lambda\left[\boldsymbol{r}_{2}(\boldsymbol{r}, \boldsymbol{p}, \chi), \boldsymbol{p}_{2}(\boldsymbol{r}, \boldsymbol{p}, \chi), \chi\right]\right\}
\end{aligned}
$$

with

$$
\begin{aligned}
& \boldsymbol{r}_{2}(\boldsymbol{r}, \boldsymbol{p}, \tau)=\boldsymbol{r}_{\mathrm{in} 2}+\int_{t_{i_{2}}}^{\tau} d \eta \frac{c \boldsymbol{p}_{2}\left[\boldsymbol{r}_{2}(\boldsymbol{r}, \boldsymbol{p}, \eta), \boldsymbol{p}_{2}(\boldsymbol{r}, \boldsymbol{p}, \eta), \eta\right]}{\sqrt{m^{2} c^{2}+\boldsymbol{p}_{2}^{2}\left[\boldsymbol{r}_{2}(\boldsymbol{r}, \boldsymbol{p}, \eta), \boldsymbol{p}_{2}(\boldsymbol{r}, \boldsymbol{p}, \eta), \eta\right]}} \\
& \boldsymbol{p}_{2}(\boldsymbol{r}, \boldsymbol{p}, \tau)=
\end{aligned}
$$

and

$$
\begin{aligned}
& \boldsymbol{r}_{2}(\boldsymbol{r}, \boldsymbol{p}, t)=\boldsymbol{r} \\
& \boldsymbol{p}_{2}(\boldsymbol{r}, \boldsymbol{p}, t)=\boldsymbol{p}
\end{aligned}
$$

and this shows clearly that the formal equivalence of the Vlasov equation to particle orbit theory can be generalized to the extension of the Vlasov equation to radiative plasmas.

However it is necessary to be careful, since the two sets of trajectories are quite different, although they coincide for the same $(\boldsymbol{r}, \boldsymbol{p})$. The second set forms a network and is larger than the first set. 